\title{
Firing Patterns in Superior Colliculus of Head-Unrestrained Monkey during Normal and Perturbed Gaze Saccades Reveal Short-Latency Feedback and a Sluggish Rostral Shift in Activity
}

\author{
Woo Young Choi and Daniel Guitton \\ Montreal Neurological Institute, McGill University, Montreal, Quebec H3A2B4, Canada
}

The superior colliculus (SC) encodes a saccade via the spatial position of an ensemble of active neurons on its motor map. Downstream circuits control muscles with the temporal code of firing frequency and duration. The moving hill hypothesis resolves the SC-tobrainstem spatiotemporal transformation (STT) enigma by proposing feedback to the SC which "pushes" a hill of activity (height = frequency) caudorostrally such that its instantaneous position encodes the angular error [gaze-position error (GPE)] between gaze and target. This mechanism, proposed for cat but controversial in primate, has not been tested in the head-unrestrained monkey. We do this here. During large $\sim 60^{\circ}$ control gaze shifts in the dark, a hill of activity began in the caudal SC and moved rostrally, but too sluggishly to encode veridical GPE. At gaze end the peak had not reached the rostral pole and only arrived there $\sim 80 \mathrm{~ms}$ later. No moving hill accompanied $\sim 20^{\circ}$ gaze shifts, in agreement with previous studies of head-fixed monkeys. To investigate feedback to the SC, we perturbed large gaze shifts producing initial and corrective gaze saccades separated by $50-800 \mathrm{~ms}$ of gaze immobility, the gaze plateau. Map activity was dramatically remodeled: the sluggishly moving hill stopped during the plateau, at the site encoding the corrective gaze saccade, thereby providing a tonic stationary spatially encoded memory signal of plateau GPE. A burst occurred before the corrective saccade. Conclusion: Feedback to map moves activity which encodes a low-pass filtered GPE signal, a process too slow to implement the STT but adequate for corrective gaze saccades.

\section{Introduction}

Gaze saccades, composed of coordinated eye-head movements, may be driven by a gaze-position error (GPE) signal (GPE = desired minus current gaze positions) produced by a comparator within a neurally encoded feedback loop (Laurutis and Robinson, 1986; Guitton and Volle, 1987; Pélisson et al., 1988, 1995; Tomlinson, 1990; Coimbra et al., 2000; Guitton et al., 2004; Choi and Guitton, 2006; Sylvestre and Cullen, 2006). Feedback assures accuracy by driving gaze until GPE $=0$, a process that compensates for variability in the trajectories of the eye-in-head and head-onbody platforms.

The primate superior colliculus (SC) is implicated in generating gaze saccades (Freedman and Sparks, 1997b; Klier et al., 2001; Corneil et al., 2002; Choi and Guitton, 2006), and an important question is whether it lies within the gaze feedback loop (Sparks, 2004). The SC encodes a specific saccade vector by the position of an ensemble of active neurons on a retinotopically encoded mo-

Received 0ct. 18, 2008; revised Feb. 20, 2009; accepted April 5, 2009.

This work was funded by the Canadian Institutes of Health Research. W.Y.C. held an award from the Fonds de la recherche en santé du Québec. We thank Drs H. L. Galiana, Christopher Pack, the anonymous reviewers, and D. P. Munoz and members of the Neuroscience Program at Queens University (Kingston, $0 N$, Canada) for critically reading and discussing earlier versions of this manuscript.

Correspondence should be addressed to Daniel Guitton, Montreal Neurological Institute, 3801 University Street, McGill University, Montreal, QC H3A2B4, Canada. E-mail: daniel.guitton@mcgill.ca.

DOI:10.1523/JNEUROSCI.5038-08.2009

Copyright $\odot 2009$ Society for Neuroscience $\quad$ 0270-6474/09/297166-15\$15.00/0 tor map (Klier et al., 2001). Downstream, this place code is converted to a time code, because reticular circuits control muscles with firing rate and duration. Solving this spatiotemporal transformation (STT) problem is critical to understand the flow of signals from cortex to eye and neck muscles.

In the head-unrestrained cat, the STT seems solved using feedback to the SC during a gaze saccade (Roucoux et al., 1980; Munoz et al., 1991; Lefèvre and Galiana, 1992; Bergeron and Guitton, 2000; Bergeron et al., 2003; Matsuo et al., 2004). If the level of firing frequency in the SC is represented as a relief map, then at gaze shift onset there is a bell-shaped "hill" of activity whose peak is centered on the appropriate site that encodes the desired saccade vector. As the gaze shift progresses, a putative feedback signal pushes the hill rostrally until it invades the rostral pole, where so-called fixation cells (SCFNs) are located, and the gaze saccade stops. The instantaneous location of peak firing encodes current GPE, a mechanism called the "moving hill" hypothesis.

In monkey, this hypothesis has been debated with mostly negative evidence (Munoz and Wurtz, 1995b; Anderson et al., 1998; Port et al., 2000; Moschovakis et al., 2001; Soetedjo et al., 2002b; Goossens and Van Opstal, 2006). However, contrary to studies in cat, attempts to verify this model have been performed in the head-fixed monkey making saccades $<30^{\circ}$. The SC encodes gaze saccades $>30^{\circ}$ that must be studied in the head-unrestrained 
monkey. Here, we investigate the moving hill and collicular feedback in the head-unrestrained monkey making large gaze shifts.

In cat, to show that the hill of activity is moved by feedback, we perturbed gaze shifts (Matsuo et al., 2004) by mechanically braking head movements, a procedure that mimics the natural occurrence of variable transient loads to the head (Laurutis and Robinson 1986; Guitton and Volle, 1987; Tomlinson, 1990; Choi and Guitton, 2006; Sylvestre and Cullen, 2006). We use the same approach here in monkey to show short-latency responses on the map to gaze trajectory perturbations and a gaze saccade amplitude-dependent moving hill.

\section{Materials and Methods}

The following three subsections have been described in more detail in Choi and Guitton (2006).

Animal preparation. Two adult male rhesus monkeys (Macaca mulatta) were anaesthetized with isoflurane and implanted with a headholding device, a scleral search coil and a unit-recording chamber over the SC. Gaze and head positions were measured with standard magnetic search-coil techniques. Surgical and experimental protocols were approved by the Animal Care Committee of the Montreal Neurological Institute and complied with the Canadian Council on Animal Care policy on the use of laboratory animals.

Experimental paradigms. The monkey's head was attached, via two universal joints and a linear bearing, to a shaft that freely rotated in a housing containing a friction clutch. This minimized restraint of the head while permitting brief immobilizations of the shaft. The animals performed, with head either fixed or unrestrained, visually guided, overlap, and memory-guided saccade tasks for a liquid reward. Target spots were front-projected by a laser beam on a black screen facing the monkey. We searched for cells using the overlap and memory-guided saccade tasks with the animal's head unrestrained. In the overlap task, the animal maintained fixation of a small light point for a random period between $700-1100 \mathrm{~ms}$. Then, the target was presented in periphery while the fixation point (FP) remained on for another randomized duration, 600 $1100 \mathrm{~ms}$ (the "overlap"). At FP off, the monkey had to make a gaze shift to the visible target. In the memory-guided saccade task (see Fig. $1 A$ ), the animal fixated FP for a random period, 700-1100 ms. Then, while FP remained on, the target was flashed for $250 \mathrm{~ms}$. After a randomized delay of 600-1100 ms, FP disappeared and the monkey had to make a gaze shift, in the dark, to the remembered location of the previously flashed target. In this paradigm, our monkeys made no corrective gaze saccade after the initial saccade. In our typical search for cells, target locations varied randomly between -20 and $20^{\circ}$ vertically and 0 and $60^{\circ}$ horizontally contralateral to the recording sites, in $5^{\circ}$ increments ( 116 random target positions). When a cell was identified as having a movement field on or close to the horizontal meridian, formal testing began. For perturbation and control trials, we used the memory-guided saccade task with target positions between 10 and $60^{\circ}$, horizontally in $10^{\circ}$ increments. Targets were typically presented in the following proportion: $1 / 3$ of presentations between 10 and $40^{\circ}$ and $1 / 3$ at $50^{\circ}$ and $1 / 3$ at $60^{\circ}$. To prevent the monkey from dark adapting, between every trial the room was lit by a fluorescent bulb with short decay time.

We randomized trials such that we had $\sim 60 \%$ control and $40 \%$ brake trials. Typical perturbed head and gaze trajectories we studied are shown in Figure $1 B$. The brake was on for random times, $20-400 \mathrm{~ms}$, and was unpredictable to the monkey. In perturbation trials, horizontal head movements were stopped by activating the friction clutch when initial gaze velocity reached $40 \%$, defined as gaze onset. We triggered the brake soon after gaze shift onset because head-immobilization occurred while head velocity was low which avoided important head decelerations and consequent loss of contact with a cell. When the gaze velocity criterion was reached, the computer generated a brake trigger pulse and the resulting brake-induced head deceleration occurred $\sim 25$ ms later which was early in a gaze shift since the fast large gaze saccades were generally $\sim 200$ $\mathrm{ms}$ in duration. In our analysis and presentation of the data, the brake effects on neural responses are referred to the time of onset of head deceleration.
Single-cell recording. Action potentials of neurons at different locations in the intermediate layers of the SC were recorded with tungsten microelectrodes which were advanced hydraulically with a remote actuator (Narishige). The topographic retinotopic organization of the SC motor map was deduced by determining the amplitude and direction of gaze shifts "preferred" by each cell. We restricted our penetrations to the vicinity of the horizontal meridian which permitted more trials at different amplitudes, in both control and perturbation trials, than if we had attempted to define a full two-dimensional movement field with oblique saccades. This was critical because the probability of losing a cell was high in perturbation trials. Furthermore, only horizontal gaze shifts could be perturbed. The location of a cell along the horizontal meridian was estimated based on the peak discharge of its one-dimensional movement field (see below).

Action potentials were converted to logic pulses via a time-amplitude window discriminator (BAK Electronics) and stored along with the head and gaze movement traces and the FP and target marker signals, using data acquisition software (REX). The movement channels were sampled at $1 \mathrm{kHz}$. We consistently verified on a storage oscilloscope that head trajectory perturbations did not add noise to our action potential channel or reduce the amplitude of action potentials so as to compromise their detection by the window discriminator.

Calculation of firing rate. Data were analyzed offline with Matlab (MathWorks). Gaze = eye-in-space $=$ eye-in-head + head-in-space. Eye-in-head was calculated by subtracting measured head from measured gaze positions. Gaze shift onset and offset were determined using 40 and $30 \%$ s gaze velocity criteria respectively. To estimate a cell's instantaneous discharge rate, a spike-density function was generated by substituting all spike events with a Gaussian pulse of width $\sigma=10 \mathrm{~ms}$ and then summing all the Gaussians together to generate a continuous function in time.

Movement field calculation. We obtained each cell's movement field by plotting mean firing frequency versus gaze shift amplitude. Mean firing frequency was calculated, for any one gaze shift by counting the number of spikes in the interval beginning $10 \mathrm{~ms}$ before gaze shift onset to $10 \mathrm{~ms}$ before its end. This gave one point in the movement field plot (see Fig. $2 A, D$; supplemental Fig. 1, available at www.jneurosci.org as supplemental material). The movement field plot of mean firing frequency versus gaze shift amplitude for each cell was quite asymmetric, falling off more rapidly with decreasing than for increasing gaze saccade size. Accordingly, for each cell we fitted the following log-Gaussian equation to its one-dimensional movement field data (Bruce and Goldberg, 1985):

$$
\begin{aligned}
& \text { Mean firing frequency }=\text { bias }+\mathrm{ff}_{\max } \\
& \qquad \times \exp \left\{-0.5 \times\left[\left(\ln \Delta G-\ln \Delta G_{0}\right) / W\right]^{2}\right\},
\end{aligned}
$$

where $\Delta G$ is gaze shift amplitude; $\Delta G_{0}$ is $\Delta G$ at peak mean firing frequency $\left(\mathrm{ff}_{\max }\right) ; W$ is a constant representative of a field's width. This equation has four free parameters, bias, $\mathrm{ff}_{\max }, \Delta G_{0}$ and $W$ which we optimized using the method of least squares.

Cell classification. Movement-related cells in the SC have been classified as burst neurons (BN) and buildup neurons (BUN) (Munoz and Wurtz, 1995a) based on (1) differences in presaccadic level of preparatory activity, BUNs $>30 \mathrm{~Hz}$, BNs $<30 \mathrm{~Hz}$; (2) differences in movement field characteristics: BUNs have open (see Fig. 2A), BNs have closed, movement fields; and (3) BUNs are recorded $0.4 \mathrm{~mm}$ deeper in the SC. This classification scheme is controversial. Anderson et al. (1998) maintained the distinction between BNs and BUNs, but they could not reliably use the level of preparatory activity as a criterion. They kept the closed (BNs) versus open (BUNs) movement field characteristics and, as a further criterion used differences in the cell's vertical location in the SC. Here, as in Anderson at al. (1998), we could not reliably group neurons based on their level of preparatory activity. Furthermore, we found the Anderson et al. (1998) criteria of depth of the cell to be unreliable since the difference is small and the most dorsal layer of the SC could not be defined reliably after a few close penetrations. For cells in the middle region of the SC- our " $13^{\circ}$ zone"-we also had difficulty in using the open versus closed movement field criterion. (This was not true for more caudal cells.) For example, the definition of open-ended movement field 
for a neuron at the $13^{\circ}$ location required that we state the minimum accepted discharge for a large say $53^{\circ}$ movement, but there was a continuum in this value (supplemental Fig. $1 B$, available at www.jneurosci.org as supplemental material). Given that we found a continuum in all properties in the $13^{\circ}$ zone we opted, for the entire map, to not use the terminology $\mathrm{BN}$ versus BUN, and limit our classification criterion to whether cells had open or closed movement fields as defined very conservatively. Thus, for a cell to be classified as having an open movement field during $53^{\circ}$ gaze shifts it needed to discharge for all available trials at least 2 spikes on average, in the period $10 \mathrm{~ms}$ before gaze onset to $10 \mathrm{~ms}$ before end. Given that the typical $53^{\circ}$ gaze shift lasted $\sim 200 \mathrm{~ms}$, this meant that the lowest firing cell that was retained in our population analysis had a mean discharge rate of $\sim 10$ spikes/s and a peak firing frequency of $\sim 15$ spikes/s. This 10 spikes/s criterion, which provided a lower bound to the mean firing rate during $53^{\circ}$ gaze shifts, needed to be applied only to cells in the $13^{\circ}$ zone because it was necessary to discard cells with zero discharge which would have been outliers in our normalization calculations (described below). Cells which had zero discharge for $53^{\circ}$ gaze shifts did not exist caudal to the $13^{\circ}$ zone. Using an even lower bound, i.e., $<10 \mathrm{~Hz}$, on mean discharge rate for cells in the $13^{\circ}$ zone would have added noise to a cell's mean discharge, due to the large influence of even an additional single spike. However, the excluded low firing $(<10 \mathrm{~Hz})$ cells, all in the $13^{\circ}$ zone, were a minority in that zone $(6 / 27)$ and changing the criterion to include a few more would not have affected the conclusions of this study that are based on a population response.

Firing frequency profiles in mid-SC. To quantify whether the level of activity of the complex firing frequency profiles in the $13^{\circ}$ zone (see Fig. $3 A$ ) was greatest at the onset or end of large $\sim 50^{\circ}$, gaze shifts, we used the firing index, $\mathrm{FI}=\left(\mathrm{ff}_{\text {gazestart }}-\mathrm{ff}_{\text {gazeend }}\right) /\left(\mathrm{ff}_{\text {gazestart }}+\mathrm{ff}_{\text {gazeend }}\right)$ (see Fig. $3 B)$, where $\mathrm{ff}_{\text {gazestart }}$ and $\mathrm{ff}_{\text {gazeend }}$ are the mean firing frequencies at gaze onset and offset, respectively. These values were obtained by aligning all the spike density plots available for a given cell on gaze onset (or gaze offset), averaging the traces and taking from the mean curve the firing frequency at the points of interest.

Brake-induced suppression of activity. Shortly after brake application near the onset of large gaze shifts, the activity of cells in the middle SC was transiently suppressed. To evaluate the level of suppression we used the suppression index $\left.=\left[\mathrm{ff}_{\text {justbefore }}-\mathrm{ff}_{+40 \mathrm{~ms}}\right] /\left[\mathrm{ff}_{\text {justbefore }}\right]\right)$, where $\mathrm{ff}_{\text {justbefore }}$ is the firing frequency just before the onset of suppression and $\mathrm{ff}_{+40 \mathrm{~ms}}$ is the firing frequency $40 \mathrm{~ms}$ later. The activity levels were obtained for a given cell by aligning all the available firing frequency profiles (spike density plots) for perturbation trials on the onset of head deceleration and measuring the required frequencies from the mean spike density profile.

Spatial distribution of map activity. To locate a cell on the SC's horizontal meridian, we followed Munoz and Wurtz (1995) and Anderson et al. (1998) and used the map equation proposed by Ottes et al. (1986):

$$
x=1.4[\ln (1+R / 3.0)],
$$

where $x$ is distance in $\mathrm{mm}$ from the rostral pole of the SC and $R$ is the distance in degrees of visual space from the fixation point. Equation 2 can be rewritten as follows:

$$
R=3.0\left(e^{x / 1.4}-1\right) .
$$

We defined a cell's location, $x_{0}$, on the horizontal meridian of the SC map by finding the gaze saccade amplitude $\Delta G_{0}$, Equation 1, corresponding to the peak of the cell's movement field.
Note that Equation 1 and its constants were obtained from a study of the head-fixed monkey, but for head-unrestrained gaze shifts, the characteristics of the caudal SC's motor map are ill defined. Indeed, for target offsets greater than $\sim 30^{\circ}$ the head contributes to the gaze displacement (Guitton and Volle, 1987; Freedman and Sparks, 1997a), and mapping studies in head-unrestrained monkeys are necessary to determine the caudal map characteristics. Unfortunately, such mapping studies are unavailable.

In Figures 7-9, we used spline fits to the spatial distribution of SC map activity at different stages of a gaze shift to show the evolution of map activity. As in Anderson et al. (1998), to test, in control trials, the significance of the different peak positions at gaze onset and offset (see Fig. $7 \mathrm{~A}$, panels 1 and 4, 7C,D), we used a bootstrap procedure to determine the $95 \%$ confidence intervals (Carpenter and Bithell, 2000). Note that contrary to Anderson et al. (1998), in our bootstrap analysis, we did not shuffle cell positions, as this would have been contradictory to the goal of demonstrating a specific spatial pattern at each GPE. Briefly, from the firing characteristics of each of the 62 cells in our population at, say, gaze onset (see Fig. $7 \mathrm{A1}$ ), we picked 62 cells at random, repeating the process 10,000 times by resampling with replacement (i.e., same cell could be used more than once in any one round of picks). A spline was fit through each new, resampled population. The $95 \%$ confidence interval was determined by discarding the lowest $2.5 \%$ and the highest $2.5 \%$ values of the ensemble of the splines at each spatial location. The $95 \%$ confidence interval in the location of the peak of activity on the map was calculated by determining its location at each iteration (hence, 10,000 reiterated peak locations in total) and discarding the lowest and highest $2.5 \%$ values.

\section{Results}

\section{General}

In two monkeys, we studied collicular discharges during headunrestrained gaze shifts to the remembered location of a previously flashed target on the horizontal plane, $10^{\circ}-60^{\circ}$ contralateral to the recording sites (Materials and Methods) (Fig. 1). Gaze shifts were made in the dark, which assured that the motorrelated discharges were not "contaminated" by visual responses. We compared discharges of cells on the SC motor map during control and perturbed gaze shifts. Figure $1 A$ shows a typical con- 
trol $50^{\circ}$ gaze saccade which displaced gaze from right $30^{\circ}$ to left $20^{\circ}$ of body centered space. After gaze saccade end, the vestibuloocular reflex (VOR) moved the eyes opposite to ongoing head motion, thereby stabilizing gaze in space.

In $40 \%$ of trials with large $50^{\circ}-60^{\circ}$ gaze shifts, head movements were unexpectedly and briefly halted for $20-400 \mathrm{~ms}$, beginning just after head-movement onset. In a typical perturbation trial (200 ms head brake) (Fig. $1 B$ ), an $\sim 35^{\circ}$ eye saccade occurred and brought the eye $\sim 15^{\circ}$ to the left of orbital center, well within the oculomotor range. Because the head was immobile the first gaze saccade was virtually the same as the eye saccade. Head-brakes caused important trajectory perturbations: the initial gaze saccade was followed by a "gaze plateau" during which the visual axis was immobile relative to space. Prolongation of the gaze plateau after head release was due to the VOR that moved the eyes opposite to head motion. The gaze plateau was terminated by an eye saccade that, together with ongoing head motion, generated a $15^{\circ}$ corrective gaze saccade that brought gaze to the remembered location of the target. The duration of the plateau depended on brake duration, duration of the first saccade, and time between brake offset (head-release) and when the corrective eye saccade was generated. Thus, the onset and offset, respectively, of a gaze plateau did not correspond exactly to the onset and offset of a head-brake. Plateau duration was generally longer than brake duration. Across all experiments, $95 \%$ of the plateau durations varied between 25 and $565 \mathrm{~ms}$; mean, $216 \pm 128 \mathrm{~ms}$ (SD); median, $203 \mathrm{~ms}$. The distribution of time between head release and onset of the corrective gaze saccade for all trials was skewed with the peak closer to brake offset: mean, $121 \pm 125 \mathrm{~ms}$; median, $81 \mathrm{~ms}$. The monkeys never generated a corrective-or any saccade-before the head was released. Very occasionally $(<3 \%$ trials), there was no saccade on head release and these trials were discarded.

For the large perturbed gaze shifts, we studied here the amplitude of the initial gaze saccade varied from trial to trial. At plateau end, the corrective gaze saccade compensated for this variability such that gaze accuracy in perturbation trials was comparable with control.

\section{Temporal properties of discharges at caudal and mid-SC locations in control trials}

We obtained sufficient data in control and head-brake perturbed gaze shifts to characterize the discharge patterns of 51 cells -37 in monkey "M1" and 14 in monkey "M2"-recorded throughout a large rostrocaudal extent of the SC motor map. We focus, in this section, on how discharges evolved with time at two example locations in the caudal and middle SC, respectively. The former is the location where activity originated in the large gaze shifts we studied here. The latter is near the locations which encoded the range of corrective gaze saccades in perturbation trials.

We consider first a population of 18 cells located on the horizontal meridian near the SC's caudal end for which we had sufficient trials in both the control and perturbation conditions. These cells had preferred horizontal gaze shift amplitudes in the range $40^{\circ}-70^{\circ}$, mean $53^{\circ}$, and based on the map of Ottes et al. (1986) were situated $>3.7 \mathrm{~mm}$ caudal to the rostral putative fixation zone. We refer to this caudal area as the $53^{\circ}$ zone. Figure $2 \mathrm{~A}$ shows the horizontal movement field (Materials and Methods) of a typical cell, M1-50, obtained in control trials covering a wide range of amplitudes and whose optimal amplitude was $\sim 53^{\circ}$. This cell's firing frequency, for any one amplitude, was noisy typical of SC motor-related discharges, and hence, we calculated all movement fields based on mean firing frequency not peak firing, at each amplitude. When testing M1-50, we did not obtain trials with amplitudes more than $\sim 60^{\circ}$, and the movement field of this cell appeared "open-ended."

In control, $50^{\circ}$ gaze shifts (Fig. $2 C$ ), cell M1-50, burst with a flat-topped bell-shaped firing frequency profile with peak instantaneous frequency at $105 \mathrm{~Hz}$ and mean of $95 \mathrm{~Hz}$. Firing frequency began increasing $\sim 200 \mathrm{~ms}$ before, and peak firing occurred $\sim 80$ ms after gaze saccade onset. At control gaze shift end, the activity had declined to $\sim 40 \%$ of its peak value. By comparison, for $15^{\circ}$ control gaze shifts, the instantaneous firing frequency profile of cell M1-50 had a low-level burst (Fig. 2B), as expected from its movement field (Fig. 2A).

We next consider a population of 27 cells located about midway on the horizontal meridian of the map between the fixation zone and the very caudal end and for which we also had sufficient trials in control and perturbation conditions. These cells had preferred horizontal gaze amplitudes of $7^{\circ}-17^{\circ}$, mean $13^{\circ}$, and based on the map of Ottes et al. (1986), were situated in the range 1.5-2.7 mm, mean $2.2 \mathrm{~mm}$ caudal to the rostral fixation zone. We will refer to this region as the $13^{\circ}$ zone. Supplemental Figure $1 B$, available at www.jneurosci.org as supplemental material, shows the horizontal movement fields of all cells in the $13^{\circ}$ zone. Figure $2 \mathrm{D}$ shows the horizontal movement field of example cell M1-15 in this zone, obtained from the mean firing frequency of individual control trials covering a wide range of horizontal amplitudes. At the optimal amplitude, the mean frequency from the movement field was $\sim 160 \mathrm{~Hz}$ (Fig. 2D). Compared with cells in the caudal SC, those near the $13^{\circ}$ location had a preferred amplitude that was defined by a sharper and clearer peak in the movement field and by a higher burst frequency. For example, for $\sim 15^{\circ}$ control gaze shifts-close to its preferred amplitude-cell M1-15 had a Gaussian-shaped firing frequency profile with peak at $\sim 215 \mathrm{~Hz}$ (Fig. 2E). The latter burst was more vigorous than that of cell M1-50 for its optimal amplitude movement (Fig. 2C).

For $\sim 50^{\circ}$ control gaze shifts, M1-15 had a mean firing frequency of $\sim 25 \mathrm{~Hz}$ (Fig. 2D). The instantaneous firing frequency profile (Fig. $2 F$ ) showed a gradually increasing discharge which began $\sim 150$ ms before gaze shift onset. There was a clear late peak $(\sim 45 \mathrm{~Hz})$, just preceding saccade end, and the suggestion of an early smaller peak $(25 \mathrm{~Hz})$ synchronous with saccade onset. (Each peak is identified by an asterisk in Fig. $2 F$, bottom histogram, see also Fig. 3A.) The twin-peak profile was a characteristic of the response to large gaze shifts of the population of cells in the $13^{\circ}$ zone, which is one reason why we chose cell M1-15 as an example cell.

An important goal of this study is to show the evolution of map activity for large, $50-70^{\circ}$, gaze shifts. To enable us to compare our results with those of previous studies we needed to normalize each cell's response, at any point during a gaze shift, to its peak discharge during that gaze shift. To do this, as explained in Material and Methods, we excluded from our analysis of population activity, any cell that discharged during $\sim 53^{\circ}$ gaze shifts, with a mean frequency $<10 \mathrm{~Hz}$ measured from $10 \mathrm{~ms}$ before gaze shift onset to $10 \mathrm{~ms}$ before offset. With this criterion, in the $13^{\circ}$ zone, $21 / 27$ cells were kept for analysis and caudal to the $13^{\circ}$ zone; all cells met the criterion and were kept for analysis.

Across the population of retained cells in the $13^{\circ}$ zone, the different discharge profiles, associated with $\sim 53^{\circ}$ gaze shifts, emphasized one or the other of the two peaks seen in cell M1-15. Typical discharge profiles are shown in Figure $3 A$. To quantify whether the level of activity was greatest at the onset or end of large gaze shifts, we used the firing index (Fig. $3 B), \mathrm{FI}=\left(\mathrm{ff}_{\text {gazestart }}\right.$ $\left.-\mathrm{ff}_{\text {gazeend }}\right) /\left(\mathrm{ff}_{\text {gazestart }}+\mathrm{ff}_{\text {gazeend }}\right)$ (Materials and Methods). $\mathrm{FI}=0$ indicates equal activity levels at start and end; FI $=+1$, early 
activity only; FI $=-1$, late activity only. Note that the index FI does not indicate whether a particular cell had one or two peaks, it just provides a nondimensional measure, for a cell, of the relative strength of activity at the start and end of a large gaze shift. Across the 21 neurons in the $13^{\circ}$ zone, FI was distributed throughout the range -0.8 to +0.5 , skewed toward negative values with a mean value $\approx-0.3$, indicating that population peak firing was shifted toward gaze end. Thus, cells like M1-15 (we recorded 15/21, the 2 columns to left of 0 ) had both early and late peaks; cells like M1-15E (3/21, two columns to right of 0 ) mainly early activity; and cells like M1-15L (3/21) mainly late activity.

Temporal properties of neural discharges at caudal and mid-SC locations in perturbation trials

We return now to Figure 2 to consider the discharge properties of the same example cells, M1-50 and M1-15, in perturbation trials.

Before the onset of a brake-induced head deceleration, which occurred very early ( $\sim 25 \mathrm{~ms}$ ) after gaze shift onset, the discharge profiles of both cells in control and perturbation trials were similar (Fig. $2 C, F$, bottom firing frequency profiles). (When comparing the spike density traces in the control and perturbation trials bear in mind that the latter had fewer trials and consequently were more "noisy" for any one plateau duration.)

Shortly after $(\sim 10-15$ ms $)$ headdeceleration, there was, for M1-50, a sharp drop of firing frequency relative to control trials that continued until initial saccade end (Fig. 2C, bottom firing frequency profile). By comparison, for M1-15, there was a brief $(\sim 50 \mathrm{~ms})$ period in which activity was transiently suppressed, as clearly seen in the raster plots and the mean frequency profile in the bottom panel of Figure $2 F$. In M1-15, the brake-induced transient suppression was striking, but this was not true for all cells in the $13^{\circ}$ zone and there was considerable variability in the level of the suppression index $=\left[\mathrm{ff}_{\text {justbefore }}-\mathrm{ff}_{+40}\right.$ $\mathrm{ms}] /\left[\mathrm{ff}_{\text {justbefore }}\right]$ (see Materials and Methods); mean index $=0.3 \pm 0.16(\mathrm{SD})$.

After the end of the initial saccade (saccade amplitude always $<40^{\circ}$ ), there was, in both example cells, a tonic discharge whose duration increased with plateau duration. For cell M1-15, the plateau-related discharge was particularly steady and was in continuity with the discharge at the end of the initial saccade. This tonic plateau discharge was a characteristic of the population and provided, on the SC map, a spatially encoded memory trace of GPE during gaze plateaus, as will be shown later in this study. for additional details.
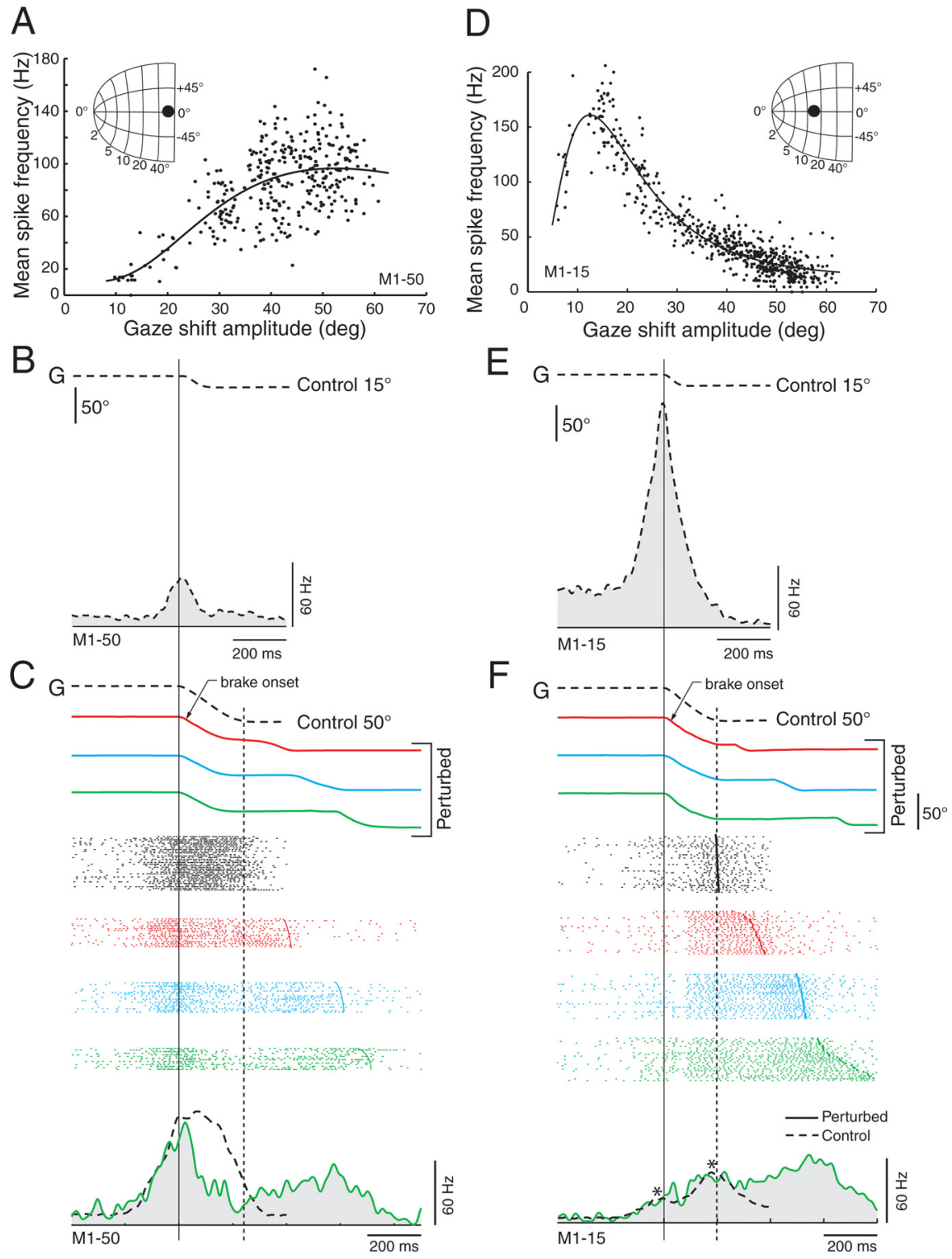

Figure 2. Discharge of cells in two representative zones $\left(53^{\circ}\right.$ and $\left.13^{\circ}\right)$ of collicular map in memory-saccade paradigm. $\boldsymbol{A}$, Movement field: mean firing frequency versus amplitude of gaze shift for example neuron (M1-50) in caudal SC (inset) which had a preferred amplitude of $\sim 50^{\circ}$. $\boldsymbol{B}$, Mean firing frequency profile (spike-density) of M1-50 showing low frequency burst that peaks on onset of $15 \pm 2.4^{\circ}(n=25)$ control gaze shift. $C$, Top, Selected examples of gaze trajectories aligned on gaze shift onset. Black dashed line, Control $\sim 50^{\circ}$ gaze shift (the cell's optimal amplitude). Colored lines, Perturbed gaze shifts ordered from top to bottom according to increasing gaze plateau duration which, itself, caused increases in overall gaze shift duration. Middle, Four rasters of action potentials, ordered from top to bottom according to increasing gaze shift duration and color-coded to match same-color gaze shifts above. Each horizontal line of a raster shows data for one trial and each tick mark identifies an action potential. Dark, near-vertical curved line at raster end indicates gaze shift end in each trial. Bottom, Spike density histogram showing firing frequency profiles for control trials (dashed line, $50.4 \pm 3.5^{\circ}, n=114$ of which 35 trials are shown in top black raster) and longest perturbed gaze shifts. D, Movement field of example neuron (M1-15) in middle SC (inset) which had a preferred amplitude of $\sim 13^{\circ}$. $\boldsymbol{E}$, High-frequency burst discharge of M1-15 during near-optimal $15 \pm 3^{\circ}(n=62)$ gaze shifts. $\boldsymbol{F}$, Discharge of M1-15 during a control and three perturbed $\sim 50^{\circ}$ gaze shifts of increasing overall durations. As in $C$, bottom panel shows control spike density histogram $\left(51 \pm 4^{\circ}, n=270\right.$, of which 34 trials shown in top-black raster) and longest perturbed gaze shift. Note double peak — each peak identified by an asterisk - firing frequency profile for large control gaze shifts and prolonged tonic firing during plateaus. Vertical solid and dotted lines in $\boldsymbol{C}$ and $\boldsymbol{F}$ indicate gaze onset and offset, respectively. See $\boldsymbol{C}$

A very short time (10 ms) after head release, the level of activity built up rapidly across the motor map, and a burst discharge occurred in both cells just before the corrective gaze saccade. This burst is blurred in Figure 2, $C$ and $F$, because the trials are not aligned on the onset of the corrective gaze saccade. They are in 
A

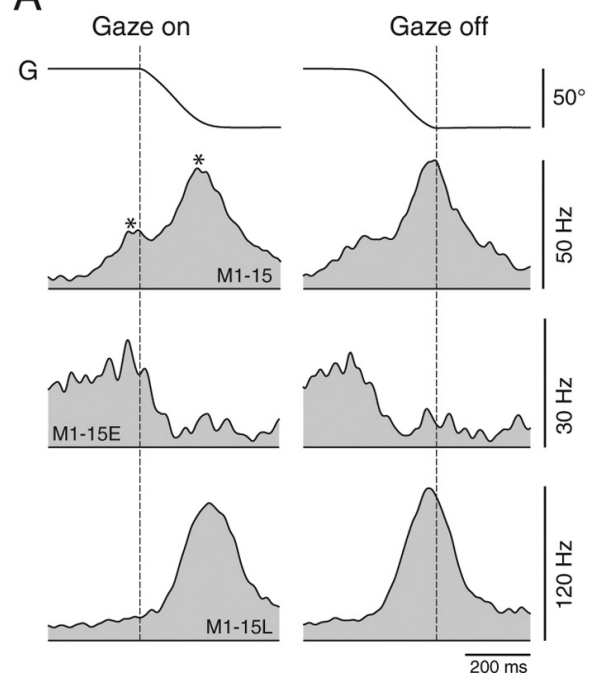

B

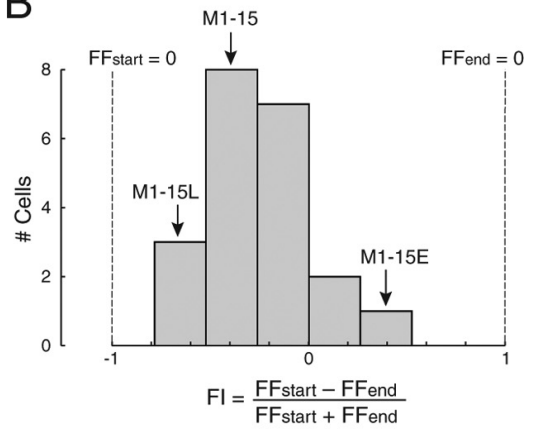

$\sim 15^{\circ}$ control gaze saccade was much stronger than the burst for the same amplitude corrective gaze saccade in perturbation trials. In general, the activity patterns in the $13^{\circ}$ zone showed that a corrective gaze saccade was not a standalone, reprogrammed, saccade, indicating that, in perturbation trials, a classic remapping of the whole SC motor map had not occurred. These effects will be considered in more detail later in relation to Figures 6-8.

\section{Dependence of plateau firing frequency on GPE}

When we perturbed large gaze shifts, there were important trial to trial differences in the amplitude and velocity of the initial eye-in-head saccade (which occurred when the head was immobile) and by extension in plateau GPE. We found no explanation for this phenomenon and we assume it was due to natural noise in the eye saccade generator which, in the natural head-unrestrained condition, is compensated due to feedback by head motion. We have seen above that cells in the $13^{\circ}$ zone discharged for large gaze shifts albeit at low frequency compared with their optimal. We found that the variability in initial saccade trajectory was reflected in the discharge of many of these cells which, after the initial saccade, led to a significant dependence of plateau firing frequency on plateau GPE. To illustrate this (Fig. 5) for two of our example cells, M1-15 and M115L (previously shown in Fig. 3A), we selected two subsets of perturbation trialseach subset with about the same plateau GPE and mean and minimum plateau durations $\approx 200 \mathrm{~ms}$ and $100 \mathrm{~ms}$, respectively-and aligned each subset for each cell on the onset and offset of plateaus and calculated the respective mean spike density histograms. The noise in the traces is due to the limited number of trials in each condition as given in Figure 5's legend.

For cell M1-15 (Fig. 5A), the temporal characteristics of the firing frequency profile were different when the plateau was located far from $\left(\mathrm{GPE}=25^{\circ}\right)$ compared with near $\left(\mathrm{GPE}=12^{\circ}\right)$ the gaze shift goal, and this property originated in the different profiles during the initial saccade, whose trajectory was affected by the brake. Thus, the initial gaze saccade was larger and faster for plateau GPE $=12^{\circ} \mathrm{com}-$ pared with $\mathrm{GPE}=25^{\circ}$. At plateau onset M1-50 is compared for both corrective (full lines) and control (dashed lines) gaze saccades of $\sim 15^{\circ}$ in amplitude, far from the optimal for this cell. In both types of gaze shifts, the firing frequency profile in each of 25 trials has been aligned on gaze saccade onset and averaged. In the perturbation trials, the irregular discharge pattern that precedes the burst reflects the sum of discharges during initial saccades and variable duration plateaus that results from averaging trials with mean and minimum plateau durations of $\sim 200 \mathrm{~ms}$ and $100 \mathrm{~ms}$, respectively. $\boldsymbol{B}$, Discharge of cell M1-15 for same conditions as M1-50. Same notation and symbols as in $\boldsymbol{A}$. The $\sim 15^{\circ}$ corrective and control gaze saccades are about optimal for this cell. Note the much lower burst frequency for corrective compared with control gaze saccades.

Figure 4 , which compares the mean spike density for $\sim 15^{\circ}$ control gaze saccades and $\sim 15^{\circ}$ corrective gaze saccades in perturbation trials. In the latter, the burst for M1-50 in control trials (Fig. $4 A$ ) was slightly smaller than in the perturbation trials wherein peak frequency rose weakly above the tonic plateau discharge. By comparison, for cell M1-15 (Fig. 4B), the burst preceding the
(Fig. $5 A$, left), firing frequency ceased increasing and, bearing in mind the "noise," tended to maintain the value it had at saccade end, and thus, the plateau firing frequency itself was greater when GPE was $12^{\circ}$ compared with $25^{\circ}$. The increase in firing frequency toward the end of the plateau was due to head release and the triggering of a corrective gaze saccade (Fig. $5 A$, right). Plateau 
firing frequency was higher for plateau $\mathrm{GPE}=12^{\circ}$ compared with $25^{\circ}$ and the correspondingly larger burst for $12^{\circ}$ corrective gaze saccades was because these are closer to M1-15's preferred amplitude.

For cells in the $13^{\circ}$ zone that had a prominent late peak and little or no early activity, of which cell M1-15L is an example (Fig. 5B), we found discharge characteristics similar to those of M1-15.

In Figure 5, $C$ and $D$, we extended these observations to a larger range of plateau GPEs. For each of the two cells, we determined subsets of trials, each with at least 5 trials at a specific plateau GPE and time, from plateau onset to head-release, long enough (minimum 100 ms, mean $200 \pm$ $91 \mathrm{~ms}$ ) to evaluate the mean plateau frequency, without including the period between head release and corrective saccade onset. The spike density traces for all trials in a subset were aligned on gaze plateau onset and a mean spike-density histogram obtained. This analysis confirmed that, for example cells M1-15 and M1-15L, plateau firing frequency varied inversely with plateau GPE (Fig. 5C,D).

We found in the $13^{\circ}$ zone a significant inverse relationship between plateau firing frequency and GPE for 11/21 cells (including M1-15 and M1-15L). The 3 early burst cells had a plateau discharge that was too low to discern a GPE dependence (e.g., cell M1-15E) (Fig. 3A; supplemental Fig. 2, available at www.jneurosci.org as supplemental material). For each of the remaining 7/21 cells, we also obtained an inverse relationship between plateau firing and GPE, but it was not significant due to a restricted data set.

In comparison to most cells in the $13^{\circ}$ zone, none of the cells in the $53^{\circ}$ zone had an observable dependence of plateau firing frequency on plateau GPE because the firing frequency after the brake declined abruptly and was low during gaze plateaus (Fig. 2C).

\section{Temporal properties of population discharges in control trials}

The population firing frequency profile for the 18 cells in the $53^{\circ}$ zone showed the expected burst that was about synchronous with the onset of $53^{\circ}$ control gaze shifts, their optimal amplitude (Fig. $6 A$ ). For small $13^{\circ}$ control gaze shifts, cells in this caudal zone showed a much smaller burst at saccade onset and no late burst occurring near saccade end. The shape of the firing frequency profile was similar for all amplitude gaze shifts. Note also the low population discharge at gaze shift end. By comparison, the population temporal discharge profile for the 21 retained cells in the $13^{\circ}$ zone (Fig. $6 \mathrm{~B}$ ) presented quite different shapes for different control gaze shift amplitudes. For $13^{\circ}$ gaze shifts, there was a sharp peak that was synchronized with gaze onset; not surprisingly, because $13^{\circ}$ is at the preferred amplitude of cells at this recording site. As gaze shift amplitude increased, the peak of the population activity in the $13^{\circ}$ zone gradually shifted toward gaze

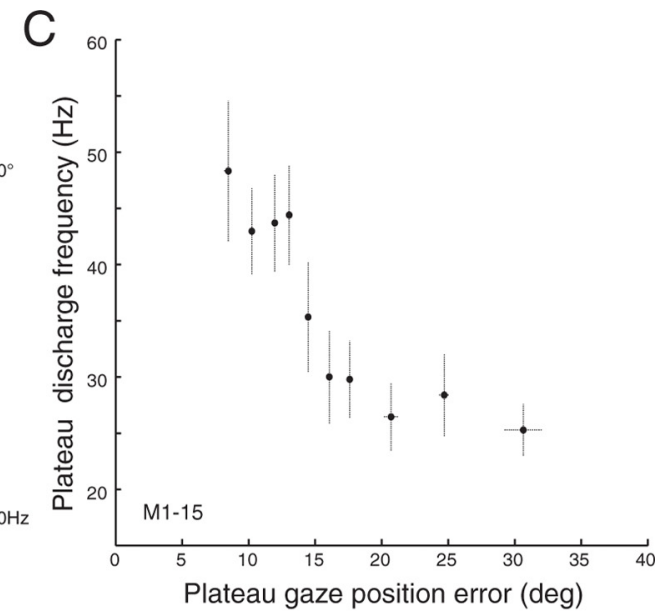

D

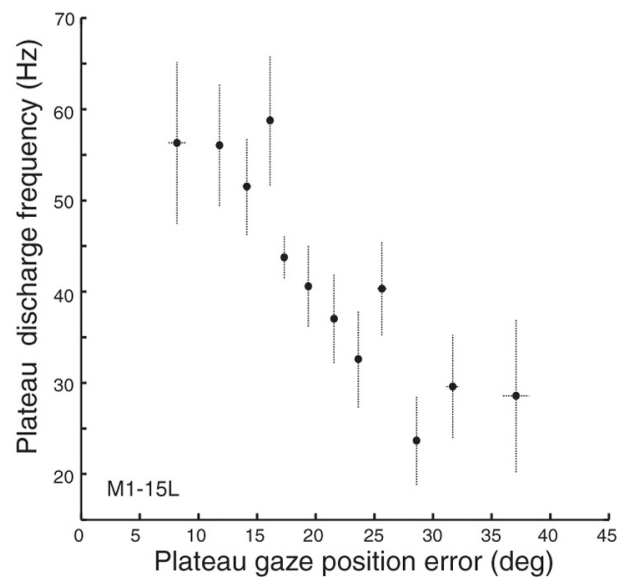

Plateau gaze position error (deg)

Figure 5. During large perturbed gaze shifts, plateau firing frequency in the $13^{\circ}$ zone decreased as plateau GPE increased. $\boldsymbol{A}$,

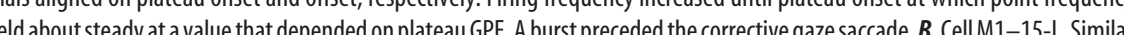
to cell M1 15 i $A$ a frequency decreased as plateau GPE increased. Error bars are SEM.

end and decreased in amplitude relative to the value for $13^{\circ}$ gaze shifts. For $50^{\circ}-60^{\circ}$ gaze shifts, the clear temporal positioning of the peak in population activity near gaze shift end is compatible with a caudorostral moving front of low-level activity, which began in the caudal SC (to be considered in Fig. 7). By comparison, for small amplitude gaze saccades, the lack of a late peak in the discharges of caudal cells rules out a rostrocaudal spread of activity (Fig. $6 \mathrm{~A}$ ). In the $13^{\circ}$ zone, the population discharge was quite high at gaze shift end, which is in line with the observations of Munoz and Wurtz (1995a) and Rodgers et al. (2006). Note also that the peak firing frequency in the $13^{\circ}$ zone for optimal $13^{\circ}$ gaze saccades was much larger that that of the population peak, in the $53^{\circ}$ zone, for optimal $53^{\circ}$ gaze saccades (compare Fig. 6A,B) [see also Freedman and Sparks (1997b) and Soetedjo et al. (2002b)].

\section{Temporal properties of population discharges in perturbation trials}

Figure $6 C$ compares the mean temporal population responses for the pooled data from the two monkeys at our example caudal $\left(\sim 53^{\circ}\right)$ and middle $\left(\sim 13^{\circ}\right)$ map locations and in the very rostral 


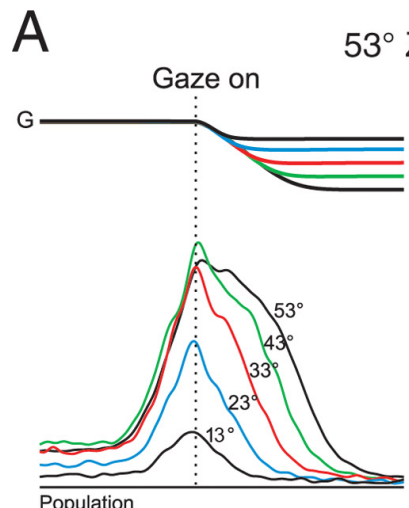

$53^{\circ}$ Zone

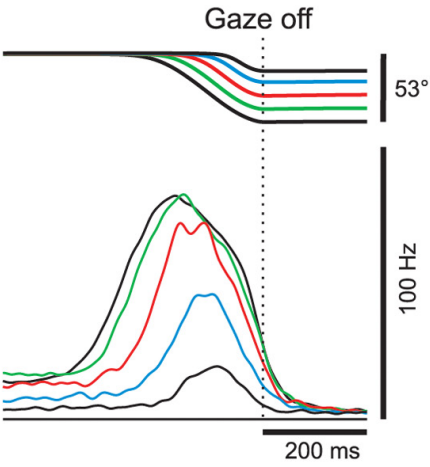

C

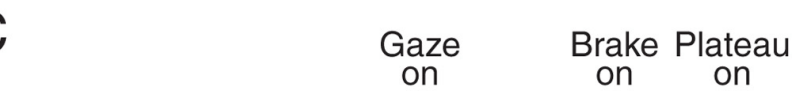

$\mathrm{B}$

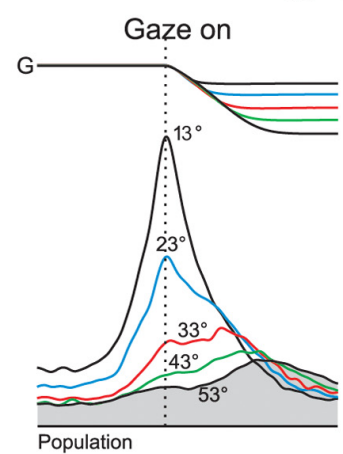

$13^{\circ}$ Zone

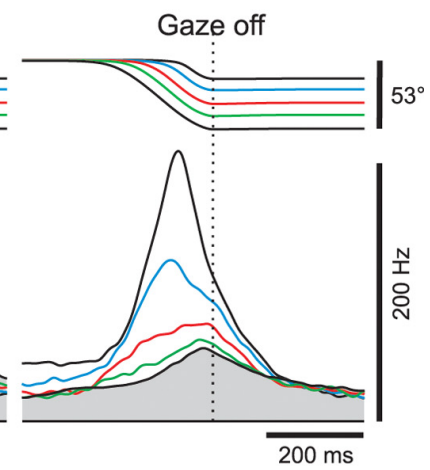

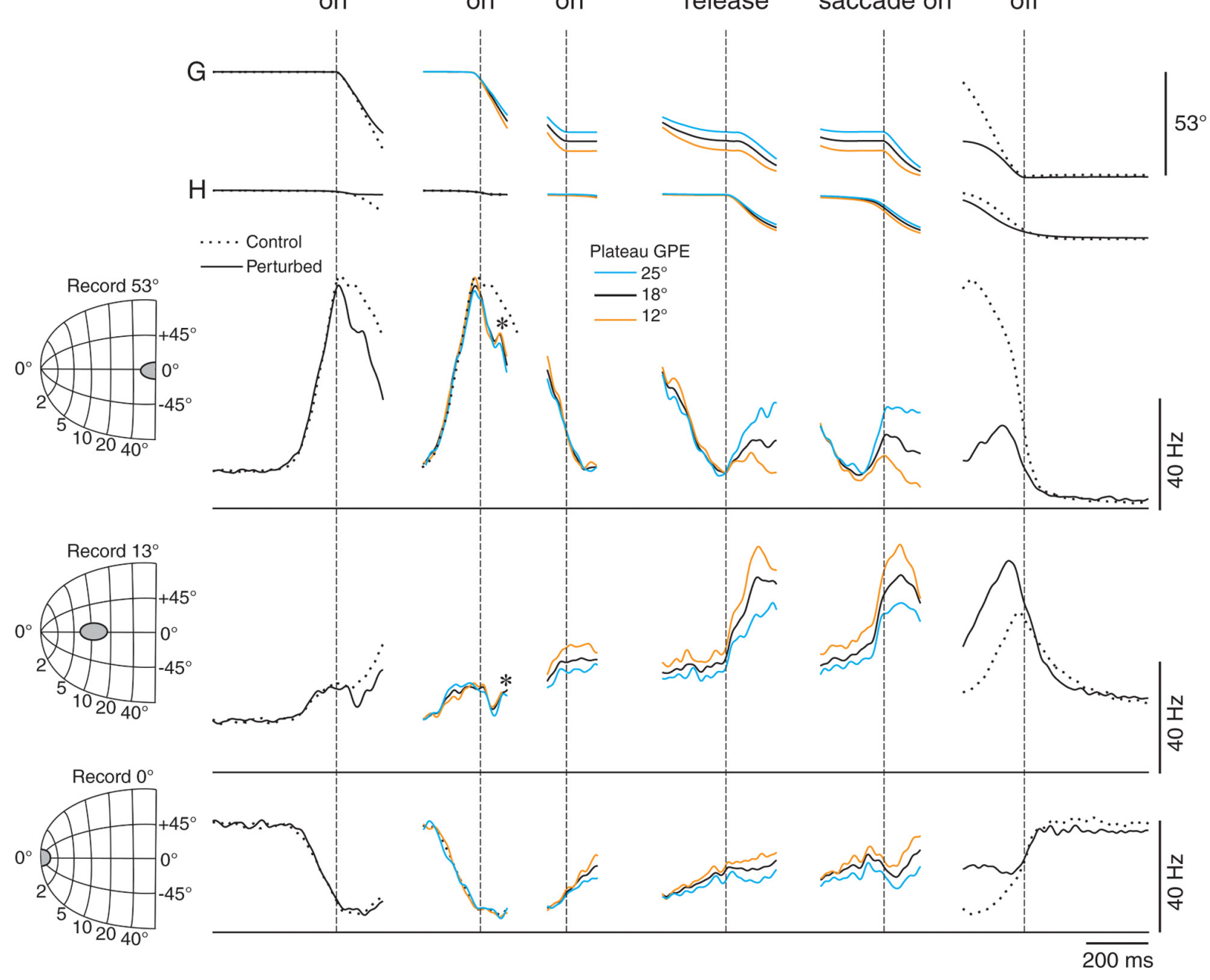

Figure 6. Overview of population temporal firing frequency profiles in the $53^{\circ}, 13^{\circ}$ and fixation zones. $A$, Population temporal firing frequency profiles for 18 cells in the caudal zone $\left(53^{\circ}\right)$ of $S C$ for control gaze shifts of different amplitudes aligned on gaze shift start (left column) and end (right column). For gaze shifts near optimal amplitude $\left(\sim 53^{\circ}\right)$ for that $\mathrm{SC}$ location, cell burst was largest and peak firing was about synchronous with gaze shift onset. As gaze shift amplitude decreased, the burst amplitude diminished, but burst profile was similar across all gaze shift amplitudes. $\boldsymbol{B}$, Population temporal firing frequency profiles for 21 cells in the $13^{\circ}$ zone for gaze shifts of different amplitudes aligned on gaze shift start (left column) and end (right column). For gaze shifts of optimal amplitude $\left(13^{\circ}\right)$, a high-frequency burst preceded saccade onset. As gaze shift amplitude increased, the early burst diminished, and a late burst became more prominent. C, Population firing frequency traces aligned on different events during control and perturbed gaze shifts in different zones of the SC. Top row, Mean discharge of 18 pooled cells, located in the $53^{\circ}$ zone of the caudal $\mathrm{SC}$, for a mean gaze shift amplitude of $53^{\circ}$. Control trials, Mean number of trials per cell $=94$, median $=83, \min =21, \max =177$. Perturbation trials, Number of trials per cell for GPE $=25^{\circ}$, mean $=20$, median $=18$; number of trials per cell for $\mathrm{GPE}=18^{\circ}$, mean $=34$, median $=30$; number of trials per cell for $\mathrm{GPE}=12^{\circ}$, mean $=21$, median $=17$. Middle row, Same for a population of 21 cells in the middle $S C\left(13^{\circ}\right.$ zone). Control trials, Mean number of trials per cell $=99$, median $=89, \min =24, \max =270$. Perturbation trials, Number of trials per cell for $\mathrm{GPE}=25^{\circ}$, mean $=$ 21 , median $=18$; number of trials per cell for $\mathrm{GPE}=18^{\circ}$, mean $=37$, median $=33$; number of trials per cell for GPE $=12^{\circ}$, mean $=23$, median $=20$. Bottom row, Same for 17 putative fixation neurons (SCFNs) located in rostral pole (taken from Choi and Guitton, 2006). From left to right, the firing frequency profiles aligned on first column, gaze shift onset; second column, brake onset; third column, gaze plateau onset; fourth column, head release; fifth column, corrective saccade onset; sixth and far right column, gaze shift end. 

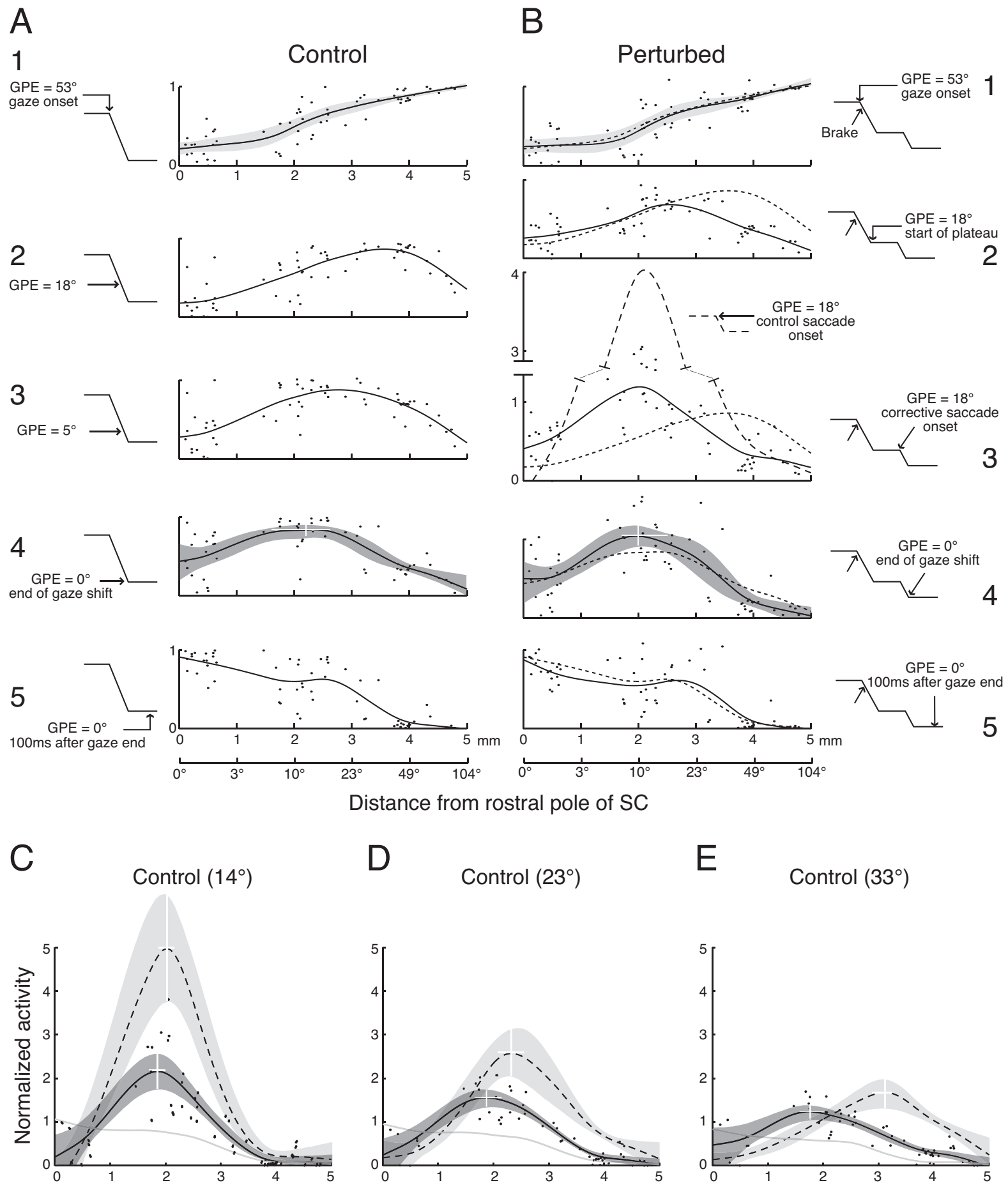

D

Control $\left(23^{\circ}\right)$
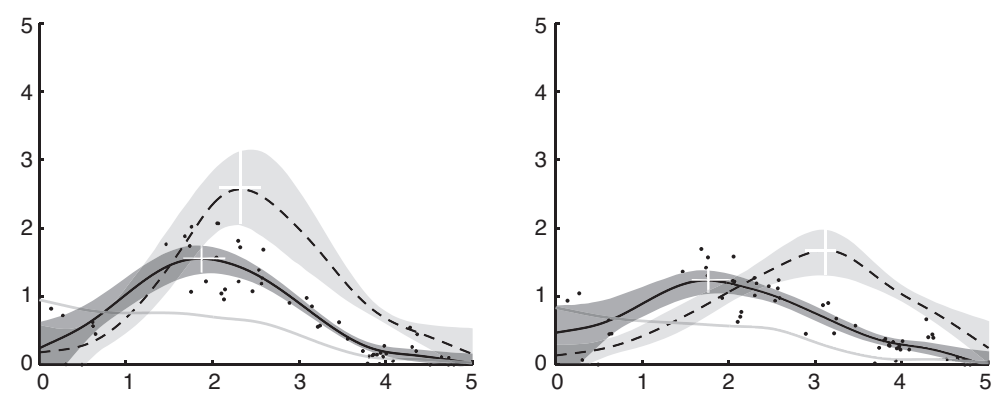

Distance from rostral pole of SC $(\mathrm{mm})$

Figure 7. Spatial distribution of normalized firing frequency across SC map at different GPEs during $53^{\circ}$ control and perturbed gaze shifts. To left and right in columns, $\boldsymbol{A}$ and $\boldsymbol{B}$ are schematics of control and perturbed gaze shifts which indicate the GPE for which data in the adjacent panel are plotted. Cells in the SC's rostral pole are SCFNs taken from Choi and Guitton (2006). A, Control (unperturbed) movement. Panels $1-5$ show activity at row $1, \mathrm{GPE}=53^{\circ}$ (gaze onset); row $2, \mathrm{GPE}=18^{\circ}$; row $3, \mathrm{GPE}=5^{\circ}$; row 4, GPE $=0^{\circ}$ (gaze shift end); and row $5,100 \mathrm{~ms}$ after gaze end. In each panel, a black dot shows the discharge of one cell at the panel's GPE, normalized to its peak firing frequency during $53^{\circ}$ control gaze shifts. Full black line is spline fit through dots (Materials and Methods). Shaded area about black line, in this and other panels, gives results of boot strap analysis showing range of possible fits within a $95 \%$ confidence interval (Materials and Methods). $\boldsymbol{B}$, Perturbation trials, Rows $\mathbf{1 - 5}$ show activity at GPEs similar to control trials. Solid black line is spline fit to perturbation data normalized as in $\boldsymbol{A}$ to the peak discharge during control $53^{\circ} \mathrm{gaze}$ shifts. Short-dashed ("dotted") line is through the control data, reproduced from the adjacent panel in $A$. Black dashed line in panel 3 shows spatial profile of discharge for a $18^{\circ}$ stand-alone control gaze saccade of same amplitude as the corrective gaze saccade in perturbation trials. Note the much higher activity in the former compared with the latter (normalized heights of peak $=4$ and 1.2 , respectively). The abscissa in $\boldsymbol{A}$ and $\boldsymbol{B}$ are given in millimeters and degrees along the SC map, calculated according to 0 ttes et al. (1986). Note that at gaze shift end $(\boldsymbol{A 4}, \boldsymbol{B} 4)$, the peak of activity had not reached the SC's rostral pole where SCFNs are located, but stopped at the $\sim 2 \mathrm{~mm}$ location. SCFNs are only reactivated $100 \mathrm{~ms}$ after gaze end. $C-E$, Map activity for control $14^{\circ}, 23^{\circ}$ and $33^{\circ}$ gaze shifts, respectively, normalized according to peak discharge during $53^{\circ}$ gaze shifts. Activity profiles at gaze start, end and $100 \mathrm{~ms}$ after gaze end are shown by dashed, full and light gray lines, respectively. Each dot is the discharge of one cell at gaze shift end, normalized to its activity during $53^{\circ}$ control gaze shifts. Note the much higher peak firing for $14^{\circ}$ compared with other gaze shifts. Peak of activity for $14^{\circ}$ gaze shifts hardly moved on the map. 
pole where SCFNs are located. For the caudal and middle zones, 18 and 21 cells, respectively, were included. To be included, a cell needed to have been recorded for a minimum of 5 trials during $\sim 53^{\circ}$ perturbed gaze shifts that had a plateau duration of at least $100 \mathrm{~ms}$ at 3 plateau GPEs $=12^{\circ}, 18^{\circ}, 25^{\circ}$. To complete the map analysis, the population activity of the 17 SCFNs in the rostral pole are taken from Choi and Guitton (2006).

The traces in Figure $6 C$ show how mean population activity evolved with time for control and perturbed gaze shifts, selected to have the same overall mean gaze shift amplitude $\left(\sim 53^{\circ}\right)$. The panels in each column were obtained by aligning the mean firing frequency profile of each cell on the relevant event (vertical dotted line) in control and perturbed gaze shifts and averaging the individual means in each condition (i.e., each cell had the same weight).

In the far left column (gaze shift onset), there is no difference between the population control and perturbation conditions, suggesting that the monkeys did not predict the condition of a particular trial. In the far right column, the mean firing frequency profile of each cell, in each condition, has been aligned on gaze shift end (for perturbed gaze shifts the alignment is on the end of corrective gaze saccades). The firing frequency profiles after gaze end are identical in control and perturbation trials, for all spatial locations, indicating that a sudden perturbation delaying the acquisition of the goal led to a concurrent equal delay in the resumption of the specific map activity associated with the end of a gaze shift.

The second column from left in Figure $6 C$ shows the temporal response of the map to head perturbations. Note first the strikingly short-latency, $<10 \mathrm{~ms}$, transient decrease in population activity after the brake-induced head declaration, in both the $13^{\circ}$ and $53^{\circ}$ zones. After brake application $(100 \mathrm{~ms})$, activity at $13^{\circ}$ had clearly "rebounded" (asterisk), while a hint of this rebound was seen (asterisk) superimposed on the overall decline of activity at the $53^{\circ}$ site.

In the third column from left, mean discharges have been aligned on plateau onset. In the $53^{\circ}$ zone, population activity that had continuously decreased during the initial saccade, kept decreasing during the initial $50 \mathrm{~ms}$ of the plateau and then remained low and independent of plateau GPE. At $13^{\circ}$ (see also Fig. 5), firing frequency abruptly ceased increasing, synchronous with plateau onset, and held constant. Plateau firing frequency increased as plateau GPE decreased, an observation similar to that made in SCFNs located further rostrally (Fig. 6C, bottom row) (Choi and Guitton, 2006). We emphasize that the variation of population plateau firing frequency with GPE was highly significant (ANOVA, $p<0.001$ ). These results indicate that the SC had been informed by feedback that the first saccade had ended but gaze was not on goal.

Some 50-80 ms after plateau onset, the map had been correctly reorganized spatially to encode a corrective gaze saccade. When the head was released (Fig. 6C, fourth column from left) and it accelerated, there were very short-latency $(<10 \mathrm{~ms})$ changes in population activity on the motor map compatible with a properly localized burst for the corrective gaze saccade. In the caudal zone, activity built-up faster for plateau GPE $=25^{\circ}$ than GPE $=12^{\circ}$ because a $25^{\circ}$ corrective gaze saccade was encoded spatially closest to the $53^{\circ}$ zone and gave the largest of the three saccade-related bursts (Fig. $6 C$, fifth column from left). The $12^{\circ}$ corrective gaze saccade itself was encoded by a burst in the $13^{\circ}$ zone, which is why at this location (Fig. 6C, fifth column from left), burst frequency was highest and lowest for GPE $=12^{\circ}$ and $25^{\circ}$, respectively, the inverse of what was seen at the $53^{\circ}$ site. For $18^{\circ}$ plateau GPE, and the associated $18^{\circ}$ corrective saccades, the activity level was between that for $\mathrm{GPE}=12^{\circ}$ and $25^{\circ}$. For SCFNs, the activity at head release decreased more for large $\left(25^{\circ}\right)$ than small $\left(12^{\circ}\right)$ corrective gaze saccades.

We noted from Figure $6 C$, second column from left, that the brake dramatically affected the firing frequency profile in the $53^{\circ}$ zone. Furthermore, the initial saccade was $<53^{\circ}$. An interesting question is whether activity during the initial saccade in perturbation trials was similar to that during a similar amplitude gaze saccade in control trials. The answer is no. To see this, for the $53^{\circ}$ zone, consider Figure $6 \mathrm{~A}$, left panel, which shows that after gaze shift onset the traces for $43^{\circ}$ and $33^{\circ}$ control gaze saccades are not coextensive and lie below the $53^{\circ}$ curve. However, Figure $6 C$, first, second and third columns from left show that the firing frequency traces in the $53^{\circ}$ zone associated with the $41^{\circ}, 35^{\circ}$ and $28^{\circ}$ initial saccades (corresponding to plateaus of $12^{\circ}, 18^{\circ}$ and $25^{\circ}$, respectively) are coextensive and amplitude invariant, although they do lie below the $53^{\circ}$ trace. This result suggests a different pattern of activity on the SC map between similar control and initial saccades resulting from gaze perturbations. More insight into this is provided in the next section.

\section{Spatial pattern of population activity on motor map for $53^{\circ}$ gaze shifts}

We now show the spatial patterns of activity on the SC map at similar GPEs during the evolution of control (Fig. 7A) and perturbed (Fig. $7 B$ ) $53^{\circ}$ gaze shifts. The rostrocaudal spatial distribution of activity was obtained by including the 45 cells we recorded on the motor map ( 18 in the $53^{\circ}$ zone, 21 in $13^{\circ}$ zone and 6 between the zones) plus the 17 SCFNs described by Choi and Guitton (2006). We included SCFNs because (1) a number of studies have suggested that there is no discontinuity between the very rostral and remaining SC map (Gandhi and Keller, 1997, 1999; Anderson et al., 1998; Krauzlis, 2005; Hafed et al., 2009), such that inclusion of SCFNs in the spatiotemporal evolution of activity provides a complete picture of map activity and (2) the very rostral SC and the remaining motor map are anatomically linked (Munoz and Istvan, 1998; Takahashi et al., 2005). However, since SCFNs were essentially silent (Choi and Guitton, 2006) until $\sim 50-80 \mathrm{~ms}$ after gaze shift end (Fig. 6C, bottom right), their activity pattern did not influence the evolution of map activity during a saccadic gaze shift but did contribute to the striking "relaxation" of map activity after gaze shift end.

To permit a comparison between the control and perturbed conditions, we plot in all panels of Figure 7, $A$ and $B$, the data from the same cells. To locate a cell we calculated the peak discharge of its movement field to obtain its preferred amplitude which was then used to assign the cell to a specific position on the SC using the map of Ottes et al. (1986) (Materials and Methods). We assumed that the SC of our monkeys conformed to the Ottes et al. (1986) map because this enabled us to compare our observations with those of Anderson et al. (1998) and Munoz and Wurtz (1995b) who also used this mapping function (Materials and Methods).

To localize SCFNs, we followed Munoz and Wurtz (1995b) (see also Anderson et al. 1998; Hafed and Krauzlis, 2008) and randomly placed them within the $0-0.7 \mathrm{~mm}$ locations on the map, which spans the rostral extent in which cells with such discharge characteristics have been recorded. For very caudal cells, the neuron's location could not usually be determined precisely because there was no clear sharp peak in the movement field plot. This uncertainty did not matter much in terms of determining the spatial profile of neural activity on the map because 
the amplitude range $60^{\circ}-85^{\circ}$ corresponds, in the very caudal SC, to the small distance range of $\sim 4.3-4.7 \mathrm{~mm}$ caudal to the rostral "fixation" zone.

During the recording of any cell, we had experimental control over neither the range of plateau GPEs nor the number of useful trials before we lost the cell, a frequent problem in these types of experiments. For perturbation trials, we needed data from cells recorded at as many different locations on the map as possible. We had the most complete data set for GPE $=18^{\circ}$; i.e., the greatest number of cells that were tested for at least 5 trials/cell with plateau duration $>100 \mathrm{~ms}$. Therefore, to compare the evolution of map activity in control and perturbed conditions, we show in Figure $7 B$ the activity pattern at this GPE in both conditions.

To generate the plots in all panels of this figure, we first normalized each cell's discharge relative to the peak value of the spike density curve in control $53^{\circ}$ gaze shifts; e.g., for cells M1-15 and $\mathrm{M} 1-15 \mathrm{~L}$ in the $13^{\circ}$ zone, the right peaks in Figure $3 \mathrm{~A}$, right panel. We then fit the spatial distribution of activity at each GPE using a cubic spline fit with the smoothing parameter fixed at 0.85 , as in Anderson et al. (1998). Note that plotting the actual activity (i.e., not-normalized) (dashed lines in supplemental Fig. 3, available at www.jneurosci.org as supplemental material) did not change the spatial distribution profile at any one GPE. This is because peak firing frequency in the caudal SC at the start of $53^{\circ}$ gaze shifts was $\sim 70 \mathrm{~Hz}$, about the same as that seen at the $2 \mathrm{~mm}$ location at gaze shift end.

At gaze shift start, the normalized map activity in control and perturbation trials (Fig. 7A1, B1) was very similar, indicating that the monkey could not predict the occurrence of either trial type. (The control and perturbation trials were independent data sets and the fact that these lines are virtually coextensive supports the reliability of our population analysis.) Not surprisingly, at the start of a $53^{\circ}$ control gaze saccade, population activity was strongest in cells of the caudal SC where normalized activity peaked at unity (Fig. 7A1,B1). The shaded area in Figure $7 A 1$ shows the $95 \%$ confidence interval on the spline fit as determined by our bootstrap analysis (Materials and Methods). As the control gaze shift proceeded, activity in the very caudal SC decreased such that at $\mathrm{GPE}=18^{\circ}$ (Fig. 7A2), the peak had moved rostrally relative to its position at gaze start, but not nearly enough to be located directly above the $18^{\circ}$ site on the map at $x \approx 2.7 \mathrm{~mm}$. At GPE $=5^{\circ}$ (Fig. $7 A 3)$, peak activity in control trials had moved further rostrally, but was still not centered at the appropriate GPE $(x \approx 1.4)$. At control gaze shift end $(\mathrm{GPE}=0)$ (Fig. 7A4), SCFNs were still inactive (Choi and Guitton, 2006), and peak activity was centered at $x=2.3 \mathrm{~mm}$. As shown by the bootstrap analysis, the $95 \%$ confidence interval of the peak's position at gaze end was between $x=1.6$ and $x=2.7$. Comparing with Figure $7 A 1$ indicates that the spatial positions of the peak at gaze shift start and end were significantly different. At gaze shift end the peak was far from the fixation zone and thus, during control gaze shifts the peak's rostral displacement was too slow to encode veridical GPE. At $\sim 100$ ms after control gaze shift end (Fig. 7A5), SCFNs were fully reactivated and the peak of activity was over the rostral pole. It is interesting that cells midway along the map, at $\sim 2.5 \mathrm{~mm}$, were still virtually as active $100 \mathrm{~ms}$ after gaze end than as at gaze end itself (see also Fig. $6 B$ ).

In $53^{\circ}$ perturbed gaze shifts, the dotted and solid lines show that the population spatial activity profile was similar at gaze saccade start to that in control trials, with peak activity located in the caudal SC (Fig. 7B1). To visualize the evolution of activity on the map, it is useful to keep in mind the temporal characteristics of the population discharge. Recall from Figure 6 that brake ap- plication induced a sharp, short-latency drop in neural firing in the caudal SC and that this decline continued until the end of the first saccade (plateau onset). Simultaneously, in the $13^{\circ}$ zone, there was a brake-induced transient suppression followed by increasing activity that continued until saccade end and stabilized, during plateaus, at values that increased as plateau GPE decreased. These temporal phenomena, transformed to the spatial domain, resulted at the end of the initial saccade (Fig. 7B2) in a substantial drop in the level of caudal activity, relative to gaze onset, in conjunction with relatively stable activity at the $2.3 \mathrm{~mm}$ location $\left(\sim 13^{\circ}\right.$ zone). The overall result was that the peak of activity had moved continuously on the map from the very caudal SC, at initial saccade onset, to the $\sim 2.6 \mathrm{~mm}$ site at initial saccade end or plateau onset (Fig. 7B2). Interestingly, there was a small rostral shift in the location of the peak of population activity between the start and end of the gaze plateau despite the immobility of the visual axis (Fig. 7, compare B2 and B3). Indeed, after plateau onset, it took $\sim 50 \mathrm{~ms}$ for the map's activity to reach a stable value and this activity pattern held steady on the map for as long as the longest $(\sim 800 \mathrm{~ms})$ plateaus we tested. Thus, from $\sim 50 \mathrm{~ms}$ after plateau onset, the plateau GPE was held steady on the map in spatial memory, using a wide bell-shaped hill of tonic activity centered on the location which encoded the corrective gaze saccade amplitude. Shortly before and during the corrective gaze saccade, there was a low-level burst of activity whose peak was at the site reached asymptotically by the rostral creep of the peak of plateau tonic memory activity. The position of peak activity in the burst accompanying the $18^{\circ}$ corrective gaze saccade remained stable during the saccade. Note that the peak firing frequency of the burst accompanying the $18^{\circ}$ corrective gaze saccades in perturbation trials was $\sim 4$ times weaker than the peak firing of control stand-alone $18^{\circ}$ gaze saccades (Fig. $7 B 3$, solid vs dashed black lines). This was also seen in Figure $4 B$. Note also, in Figure 7, $B 3$ and $B 4$, that the mean position of the peak is at $x=$ $2.0 \mathrm{~mm}\left(\equiv 10^{\circ}\right)$, with the caudal limit to the confidence interval for the peak at $x=2.6 \mathrm{~mm}\left(\equiv 16.2^{\circ}\right)$. This is not at the correct map location, in degrees, to encode the $18^{\circ}$ corrective gaze saccade, which suggests that the map equation for our monkeys may be a little different from that proposed by Ottes et al. (1986).

At the end of the corrective gaze saccade (Fig. 7B4), the peak of activity was at a significantly more rostral position than that at gaze start, but the peak had not invaded the rostral pole, as expected, because SCFNs were still relatively silent at this point. The peak arrived on the map's "zero" location only when SCFNs were fully reactivated, $\sim 80 \mathrm{~ms}$ after gaze shift end.

It is striking that the activity patterns on the map were very different between the control and perturbed conditions beginning just after brake onset and lasting at least until the end of the corrective gaze saccade. Throughout most of a perturbed gaze shift, the peak of activity was located more rostrally than for the equivalent GPE in control trials. It is only at the end of perturbed and control gaze trajectories (Fig. 7B4), and $100 \mathrm{~ms}$ after (Fig. $7 B 5$ ), that the spatial patterns in both conditions gave the same overall spatial distribution.

To compare with the above description of $53^{\circ}$ gaze shifts, we now consider in Figure $7 C-E$ the evolution of map activity between the start (dashed line), end (solid line) and $100 \mathrm{~ms}$ after the end (gray line) of $14^{\circ}, 23^{\circ}$ and $33^{\circ}$ control gaze shifts, respectively. Note that the peak of activity at the end of $33^{\circ}$ gaze shifts (Fig. 7E) was more rostral than the peak at start but that this difference became smaller as gaze shift amplitude decreased such that for $14^{\circ}$ saccades, the "gaze-start" and "gaze-end" peaks were similarly located (Fig. 7C). (The temporal evolution of activity during 

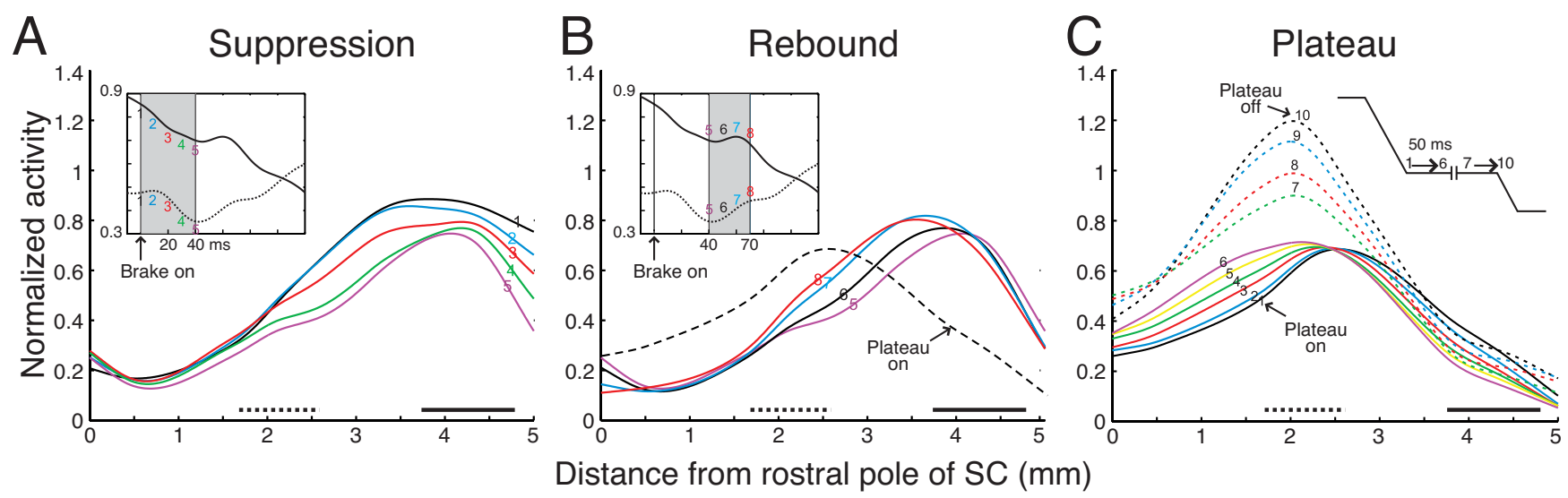

Figure 8. Spatial evolution of map activity between brake application and onset of corrective gaze saccade. $A$, Evolution of map activity, normalized relative to peak discharge in $53^{\circ}$ gaze shifts, during a $40 \mathrm{~ms}$ period beginning at brake onset. Same format as in Figure 7. Each numbered line shows the spatial firing frequency profile at $10 \mathrm{~ms}$ intervals beginning at brake onset (line 1). Inset shows temporal evolution of activity on the map at two representative locations marked by horizontal dotted and full lines just over abscissa. Bottom activity profile in inset corresponds to former. Numbers on temporal plots in inset correspond to same numbers on lines in spatial plot. $\boldsymbol{B}$, Same format as in $\boldsymbol{A}$ but in period $40-70 \mathrm{~ms}$ after brake onset. $\boldsymbol{C}$, Spatial evolution of activity in $50 \mathrm{~ms}$ period after plateau onset (lines 1-6, peak migrated rostrally) and in 50 ms period preceding corrective gaze saccade onset (lines 7-12); see inset. Map coordinates in millimeters calculated from 0ttes et al. (1986).

these $14^{\circ}, 23^{\circ}$ and $33^{\circ}$ control gaze shifts is shown in the insets of supplemental Fig. 4, available at www.jneurosci.org as supplemental material.) Our bootstrap analysis shows that there was a significant rostral displacement of the peak for $33^{\circ}$ gaze shifts but not for $12^{\circ}$ and $14^{\circ}$.

Note in Figure 7 that we did not record cells in the zone $0.7-1.4 \mathrm{~mm}\left(\sim 2^{\circ}-5^{\circ}\right.$ locations $)$, and, in any one condition, this "hole" could have resulted in a local spatial activity profile that misrepresented the true one. For example, at gaze shift end, in both control and perturbation trials, peak activity could have been located more rostrally, say over the $0.7 \mathrm{~mm}$ location. We believe that this is highly unlikely because our spatial activity profiles at the onset and end of $23^{\circ}$ gaze shifts were very similar to those of Anderson et al. (1998) who recorded many neurons in the $0.7-1.7 \mathrm{~mm}$ zone for head-fixed saccades of this amplitude (see their Fig. 8 and our supplemental Fig. 4C,D, available at www.jneurosci.org as supplemental material). This is additional strong support for the reliability of our data and its analysis.

\section{Rostrocaudal differences in firing frequency of control saccades}

We have seen that for optimal amplitude control gaze saccades, the peak firing frequency of cells in the $13^{\circ}$ zone was much higher than that in the caudal zone (Fig. 7B3). During small saccades, the major dynamic phenomenon in the rostral motor map was stationary activity and decreasing peak, while caudally it was moving activity with fixed peak. These interesting rostrocaudal differences in topographic encoding of gaze saccade amplitude may be related to the observation that head motion contributes to gaze shifts more than $\sim 20^{\circ}$ (Freedman and Sparks, 1997a) and may require feedback control.

\section{Details of map activity at brake, plateau, and corrective saccade onsets}

We now consider, in finer detail, the changes in the spatial distribution of map activity in perturbation trials over a $70 \mathrm{~ms}$ period beginning at brake onset. Shortly, $\sim 10 \mathrm{~ms}$, after a brake-induced head deceleration, and for the next $30 \mathrm{~ms}$, the map activity decreased substantially relative to control in the entire region caudal to $\sim 1.6 \mathrm{~mm}$ (Fig. $8 A$, traces $1-5$ ). In the next $30 \mathrm{~ms}$, map activity rebounded in the region $2-4 \mathrm{~mm}$ while remaining low caudal to $4 \mathrm{~mm}$ (Fig. $8 B$, traces $5-8$ ). Note that in Figure $8, A$ and $B$, brake duration was $>100 \mathrm{~ms}$, and therefore, the changes discussed above in relation to traces $1-8$ occurred while the brake was on, and therefore, the head was immobile and the initial eye saccade was ongoing. As the initial saccade progressed further, peak activity moved rostrally, and in the caudal SC decreased, to yield, at initial saccade end (三 plateau onset) a peak over the 2.6 mm location (Fig. 8 B, also shown by solid line in Fig. 7B2).

Figure $8 C$ shows evolving map activity for the first $50 \mathrm{~ms}$ after plateau onset (traces 1-6) and the last $30 \mathrm{~ms}$ preceding its end (traces 7-10), the latter coinciding with a burst discharge encoding the corrective gaze saccade. At plateau onset, the peak of activity maintained its activity level constant but kept moving rostrally on the map for $\sim 50 \mathrm{~ms}$, although gaze was immobile. Tonic activity then remained stable spatially during the entire remaining plateau (Fig. 6C) and represented a spatially encoded memory signal of plateau GPE, corresponding to the amplitude of the corrective saccade. (Fig. $2 F$ showed, for cell M1-15, that plateau activity could remain stable for $\sim 500 \mathrm{~ms}$, and we found in some trials, for M1-15 and other cells, tonic discharge continuing for $\sim 800 \mathrm{~ms}$ duration plateaus.) About $10 \mathrm{~ms}$ after head release, activity increased, first slowly then more rapidly leading to the small burst that accompanied the corrective gaze saccade.

\section{Does the SC encode a damped representation of the real gaze trajectory?}

We found for both large control and perturbed gaze shifts that the position of the peak of activity shifted rostrally but never encoded veridical GPE, except in perturbation trials, at $\sim 50 \mathrm{~ms}$ after plateau onset. Furthermore, we found little or no rostral movement of the peak discharge during control gaze shifts of amplitudes $\sim<25^{\circ}$. These diverse observations may be reconciled under the one hypothesis that activity on the SC map encoded a damped or filtered version of the real gaze shift. Indeed, in Choi and Guitton (2006), we found that SCFNs were modulated by a first-order filtered GPE with the time constant of the filter $\tau \approx 100 \mathrm{~ms}$. Given the anatomical links between the rostral pole and the motor map, it is logical to apply the same model to the motor map (Fig. 9A). To evaluate this hypothesis, we repeated the calculations leading to Figure $7 A$, but this time we plotted (Fig. 9B, solid line) the population discharge on the map versus the GPE of a hypotheti- 
cal $53^{\circ}$ gaze shift whose trajectory was calculated by filtering a real $53^{\circ}$ control gaze shift with $\tau=100 \mathrm{~ms}$. This analysis shows that the moving peak of the population firing frequency profile encoded well the filtered GPE $\left(\mathrm{GPE}_{\mathrm{f}}\right)$ of the $53^{\circ}$ control gaze saccade, whereas, as we saw above, map activity did not encode the veridical (unfiltered) GPE (Fig. 9B, dashed line, same as solid line in Fig. $7 A$ ). The peak of activity at the end of the filtered trajectory was located in the rostral pole, because SCFNs are fully reactivated only $\sim 50-100 \mathrm{~ms}$ after the end of a real gaze shift (Choi and Guitton 2006). The filter model also accounts for the observation, in large perturbed movements, that the peak of activity kept moving for a short time on the map during the initial portion of gaze plateaus. This was because the filtered trajectory of the initial gaze saccade was still moving when the real initial saccade had ended.

Note, importantly, that map activity during a filtered control $14^{\circ}$ gaze shift did not, as in the real trajectory, move rostrally (Fig. 9C). This was because the duration of small saccades was $<100 \mathrm{~ms}$ such that when the real saccade was over its filtered version had not progressed much.

During the presentation of Figure 7, the reader may have wondered why the peak of activity in control trials ended at about the same location, $x \approx 2 \mathrm{~mm}$ (Fig. 7A4), as the peak of activity preceding the corrective gaze saccade in perturbation trials (Fig. 7B3). The filtered GPE hypothesis implies that this is fortuitous. Indeed, had the chosen plateau GPE in perturbation trials not been $18^{\circ}$ but rather say $30^{\circ}$ (or $12^{\circ}$ ) the peak before the corrective gaze saccade would have been at $x>2 \mathrm{~mm}$ (or $<2 \mathrm{~mm}$ ).

\section{Discussion}

\section{Feedback to the SC}

We have presented the first evidence of short-latency intrasaccadic feedback to the primate SC obtained in a task that mimics natural conditions. Our observations require a reappraisal of the classic hypothesis (Moschovakis et al., 1996; Scudder et al., 2002; Goossens and Van Opstal, 2006) that, in monkey, the SC's output for all saccades is a predetermined "ballistic" motor command emitted open-loop and sent to brainstem eye-head motor circuits from a fixed locus on the SC map. Indeed, during large control gaze shifts, the locus of map activity did not remain immobile in the caudal SC. Furthermore, this pattern was quickly remodeled in perturbation trials. The profound rapid response of the temporal firing frequency profile at different positions on the map, after perturbations to large gaze shifts, is strong evidence for feedback. Indeed, shortly ( $\sim 10 \mathrm{~ms})$ after the onset of head-deceleration, cells throughout the motor map decreased their firing frequency. In the $13^{\circ}$ zone-a site close

Figure 9. SC map may encode the spatial representation of a low-pass filtered gaze shift with time constant $\sim 100 \mathrm{~ms}$. $\boldsymbol{A}$, Schematic of proposed filter on GPE signal that we showed in Choi and Guitton (2006) modulates SCFN discharges. We suggest here that this filter also modulates discharges on motor map. GPE is obtained by subtracting actual from desired gaze shift (n) gaze shift, same as solid black line in Figure 7A. Solid line, Spline fit through black dots. Each dot is the discharge of a cell (normalized to its peak firing frequency for $53^{\circ}$ control gaze shifts) at specific GPE $s$ in the filtered $53^{\circ}$ gaze trajectory. The top

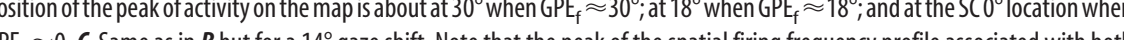
$\mathrm{GPE}_{\mathrm{f}} \approx 0$. C, Same as in $\boldsymbol{B}$ but for a $14^{\circ}$ gaze shift. Note that the peat filtered and actual $14^{\circ}$ gaze trajectories moves little on map throughout most of the gaze shift. By comparison the activity profile of the filtered $14^{\circ}$ gaze shift diminishes more rapidly with GPE than the actual.
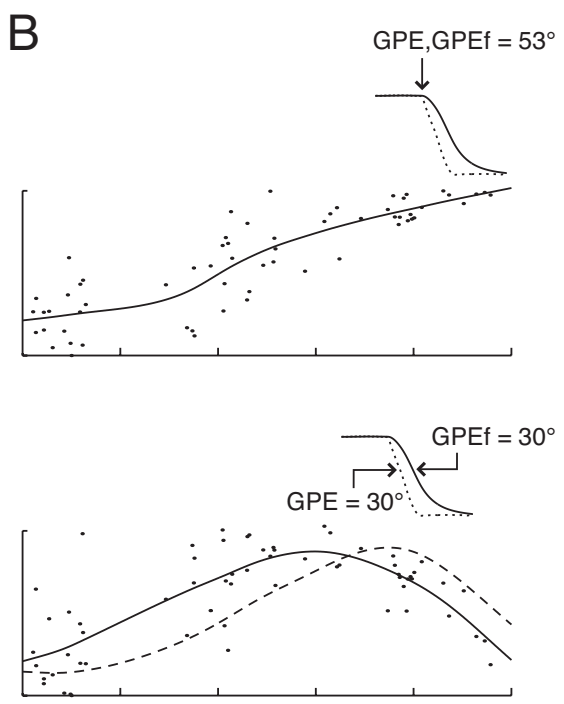

$10^{\circ}$

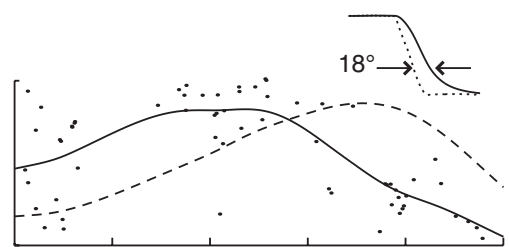

$5^{\circ}$

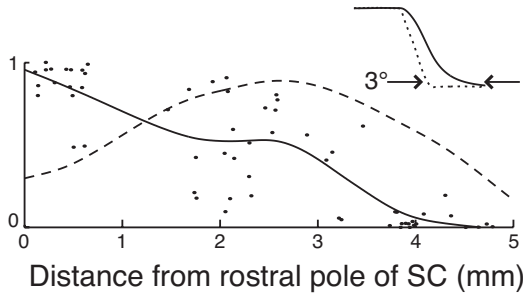

Distance from rostral pole of SC $(\mathrm{mm})$
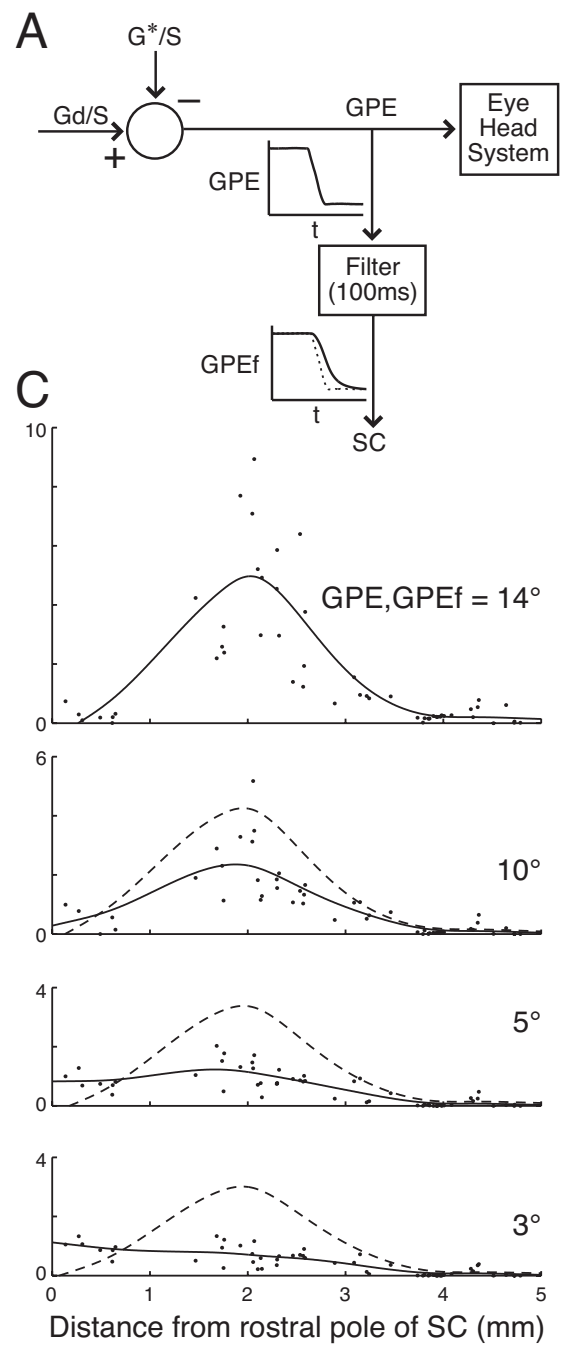

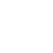


saccade; i.e., the map was "informed” about remaining GPE during the perturbation. Plateau firing frequency at the $13^{\circ}$ location depended on plateau GPE (Figs. 5, 6C), because the peak stabilized at different locations depending on corrective saccade amplitude.

Further evidence for feedback was that (1) no corrective saccades occurred until brake release, but following it there was a short-latency $(\sim 10 \mathrm{~ms})$ increase in activity leading to a burst at the site encoding the corrective gaze saccade (Figs. $6 C, 7 B, 8 C$ ). (2) At gaze shift end, map activity was the same in control and perturbed trials, no matter the duration of the perturbation.

Such map responses to random perturbations and trajectory changes could arise from feedback projections from vestibularprepositus, trigeminal and neck muscle circuits (for review, see Guitton et al., 2004). Collicular head-movement related neurons (Walton et al., 2007) were unlikely involved because they are not the "gaze-related" cells studied here. The brake's click sound might have triggered a transient collicular response, but the $\sim 10$ $\mathrm{ms}$ response latency was much shorter than that previously described for SC responses to auditory stimuli (mean, $44.8 \mathrm{~ms}$ ) (Jay and Sparks, 1987).

We could not determine if the decay of stationary activity during $<25^{\circ}$ control saccades was due to feedback because we could not perturb them. Perhaps feedback delays, coupled to the map's damped response (Fig. 9), resulted in fast small gaze shifts_composed of eye-only saccades_being controlled open-loop. This would be inline with studies that have focused on saccades $<30^{\circ}$ and concluded on open-loop control by a fixed site on the SC (for review, see Goossens and Van Ostal, 2006).

Although perturbations revealed feedback to the map, there remains the possibility that large control unperturbed gaze shifts were made without feedback control. Indeed, the clear difference between the spatial activity profiles at the start and end of large control gaze shifts (Fig. 7A1,A4) could have been due to intrinsic SC circuitry that caused the hill of activity to move rostrally. We think this is very unlikely because (1) the velocity and duration of natural gaze shifts is under voluntary control (Guitton and Volle, 1987), which would imply, if there is no feedback to the SC, voluntary control on the dynamics of the map's intrinsic circuitry, a less parsimonious mechanism than feedback which controls any trajectory; and (2) fixation neurons (SCFNs) (Choi and Guitton, 2006), which are reciprocally linked to the motor map, show a pause in activity that is tightly linked to the duration of a gaze shift regardless of whether it is a control, minimally decelerated, or completely immobilized for random times as here. Hence, our results suggest that motor map activity always reflects gaze shift kinematics, a property most parsimoniously implemented by feedback.

Our results support the models of Quaia et al. (1999) and Soetedjo et al. (2002a) that feedback to the SC does not control accurate gaze trajectories online. However, we differ in that the monkey SC does receive feedback that monitors natural perturbations of ongoing gaze trajectories to permit appropriate motor responses.

\section{Comparison with studies of perturbed eye saccades}

Activity in the SC of the head-fixed monkey has been studied during saccades interrupted by stimulation of either brainstem (Keller and Edelman, 1994; Keller et al., 2000) or rostral SC (Munoz et al., 1996). Comparisons with the present study are difficult because previous experiments (1) stopped saccades in-flight, while our head-brakes permitted the initial eye saccade to pro- ceed without an accompanying head movement and gaze shifts were interrupted only after the initial saccade was completed and (2) focused on saccades $<25^{\circ}$ while we interrupted only large gaze shifts. Only Munoz et al. (1996) provided examples of two BUNs recorded rostral to $20^{\circ}$ during large $\left(55^{\circ}\right)$ interrupted saccades. BUN activity was suppressed during stimulation but returned and held steady during the ensuing period of eye immobility before corrective saccade onset, similar to our tonic plateau activity. They did not measure, as here, whether plateau firing frequency encoded GPE. In Munoz et al. (1996), the burst discharge that preceded a corrective saccade attained control values when the interruption period was more than $\sim 100 \mathrm{~ms}$, implying that the initial motor program had been aborted. Here, the burst for the corrective saccade was always much smaller than control value (Figs. 2, 4, 7B3), implying that the initial motor program was never aborted, and our corrective gaze saccades were never "fresh" small stand-alone movements no matter plateau duration.

Saccades have also been perturbed by air-puff induced blinks (for review, see Goossens and Van Opstal, 2006). They focused on saccades $<30^{\circ}$ and concluded that the SC was "unaware" of the perturbation; it did not receive feedback. Our lack of a moving hill for small saccades is in line with their conclusion.

\section{The moving hill phenomenon}

As reviewed in the Introduction, a critical problem is how the STT is implemented. The moving hill hypothesis resolves the STT problem by placing the SC within the loop.

Evidence for an intrasaccade-related spread of activity across the primate SC is controversial but has only been studied in the head-fixed monkey. Port et al. (2000) found a caudorostral spread of activity, but it went faster than the saccade itself and arrived in the rostral SC too early to stop saccades. Munoz and Wurtz (1995b) proposed an intrasaccade caudorostral "moving front" of activity across the BUN population. A moving front was not evident for saccades $\sim<30^{\circ}$, and this was corroborated by Anderson et al. (1998), Goossens and Van Opstal (2006), and Soetedjo et al. (2002b) in the head-fixed monkey and here in the head-unrestrained monkey. Indeed, our spatially stationary distribution of map activity, at the onset and end of $\sim 23^{\circ}$ gaze saccades, is remarkably similar to that of Anderson et al. (1998) (supplemental Fig. 4, available at www.jneurosci.org as supplemental material). Furthermore, forward motion of the peak was small for $30^{\circ}$ saccades which explains why Moschovakis et al. (2001) and Goossens and Van Opstal (2006), the former using functional imaging, could not detect spreading activity for saccades of this amplitude. Given this agreement between 5 independent studies, we believe that rostral movement of peak activity in monkey is not measurable for saccades $\sim<25^{\circ}$ (there was never caudally moving activity) (Fig. $7 C-E$ ). In Munoz and Wurtz (1995), their Figure 4 shows a moving peak for large $50^{\circ}$ head-fixed eye saccades, a result we agree with. This suggests moving activity is determined by the size of a saccade, not by whether it is made head-fixed or head-unrestrained.

In the head-unrestrained cat, tectoreticular neurons carry the moving hill (Munoz et al., 1991; Bergeron et al., 2003; Guitton et al., 2004; Matsuo et al., 2004). Our map discharges were also probably efferent signals because their temporal discharge properties were similar to those of tectoreticular neurons in the headfixed monkey (Rodgers et al., 2006). However, unlike cat, the peak of moving activity in monkey did not encode the veridical instantaneous GPE.

To investigate differences in map activity between large and 
small gaze saccades using a different analysis method, we used the timing criterion of Soetedjo et al. (2002b) and found evidence for moving activity during large, not small, gaze saccades (supplemental Fig. 5, available at www.jneurosci.org as supplemental material). This confirms our assertion that previous research in the head-fixed monkey studied saccades too small to reveal a moving hill.

\section{References}

Anderson RW, Keller EL, Gandhi NJ, Das S (1998) Two dimensional saccade related population activity in superior colliculus in monkey. J Neurophysiol 80:798-817.

Bergeron A, Guitton D (2000) Fixation neurons in the superior colliculus encode distance between current and desired gaze positions. Nat Neurosci 3:932-939.

Bergeron A, Matsuo S, Guitton D (2003) Superior colliculus encodes distance to target, not saccade amplitude, in multi-step gaze shifts. Nat Neurosci 6:404-413.

Bruce CJ, Goldberg ME (1985) Primate frontal eye fields. I. Single neurons discharging before saccades. J Neurophysiol 53:603-635.

Carpenter J, Bithell J (2000) Bootstrap confidence intervals: when, which, what? A practical guide for medical statisticians. Stat Med 19:1141-1164.

Choi WY, Guitton D (2006) Responses of collicular fixation neurons to gaze shift perturbations in head-unrestrained monkey reveal gaze feedback control. Neuron 50:491-505.

Coimbra AJ, Lefèvre P, Missal M, Olivier E (2000) Difference between visually and electrically evoked gaze saccades disclosed by altering the head moment of inertia. J Neurophysiol 83:1103-1107.

Corneil BD, Olivier E, Munoz DP (2002) Neck muscle responses to stimulation of monkey superior colliculus. II. Gaze shift initiation and volitional head movements. J Neurophysiol 88:2000-2018.

Freedman EG, Sparks DL (1997a) Eye-head coordination during headunrestrained gaze shifts in rhesus monkeys. J Neurophysiol 77:2328-2348.

Freedman EG, Sparks DL (1997b) Activity of cells in the deeper layers of the superior colliculus of the rhesus monkey: evidence for a gaze displacement command. J Neurophysiol 78:1669-1690.

Gandhi NJ, Keller EL (1997) Spatial distribution and discharge characteristics of superior colliculus neurons antidromically activated rom the omnipause region in monkey. J Neurophysiol 78:2221-2215.

Gandhi NJ, Keller EL (1999) Comparison of saccades perturbed by stimulation of the rostral superior colliculus, and the omnipause neuron region. J Neurophysiol 82:3236-3253.

Goossens HH, Van Opstal AJ (2006) Dynamic ensemble coding of saccades in the monkey superior colliculus. J Neurophysiol 95:2326-2341.

Guitton D, Volle M (1987) Gaze control in humans: eye-head coordination during orienting movements to targets within and beyond the oculomotor range. J Neurophysiol 58:427-459.

Guitton D, Bergeron A, Choi WY (2004) On the role of subcortical feedback mechanisms in the control of head-unrestrained gaze saccades. In: The superior colliculus: new approaches for studying sensorimotor integration (Hall WC, Moschovakis A, eds), pp 241-275. Boca Raton, FL: CRC.

Hafed ZM, Krauzlis RJ (2008) Goal representations dominate superior colliculus activity during extrafoveal tracking. J Neurosci 28:9426-9439.

Hafed ZM, Goffart L, Krauzlis RJ (2009) A neural mechanism of microsaccade generation in the primate superior colliculus. Science 323:940-943.

Jay MF, Sparks DL (1987) Sensorimotor integration in the primate superior colliculus. II. Coordinates of auditory signals. J Neurophysiol 57:35-55.

Keller EL, Edelman JA (1994) Use of interrupted saccade paradigm to study spatial and temporal dynamics of saccadic burst cells in superior colliculus in monkey. J Neurophysiol 72:2754-2770.

Keller EL, Gandhi NJ, Vijay Sekaran S (2000) Activity in deep intermediate layer collicular neurons during interrupted saccades. Exp Brain Res 130:227-237

Klier EM, Wang H, Crawford JD (2001) The superior colliculus encodes gaze commands in retinal coordinates. Nat Neurosci 4:627-632.

Krauzlis RJ (2005) The control of voluntary eye movements: new perspectives. Neuroscientist 11:124-137.

Laurutis VP, Robinson DA (1986) The vestibulo-ocular reflex during human saccadic eye movements. J Physiol 373:209-233.
Lefèvre P, Galiana HL (1992) Dynamic feedback to the superior colliculus in a neural network model of the gaze control system. Neural Netw 5:871-890.

Matsuo S, Bergeron A, Guitton D (2004) Evidence for gaze feedback to the cat superior colliculus: discharges reflect gaze trajectory perturbations. J Neurosci 24:2760-2773.

Moschovakis AK, Scudder CA, Highstein SM (1996) The microscopic anatomy and physiology of the mammalian saccadic system. Prog Neurobiol 50:133-254.

Moschovakis AK, Gregoriou GG, Savaki HE (2001) Functional imaging of the primate superior colliculus during saccades to visual targets. Nat Neurosci 4:1026-1031.

Munoz DP, Istvan PJ (1998) Lateral inhibitory interactions in the intermediate layers of the monkey superior colliculus. J Neurophysiol 79:1193-1209.

Munoz DP, Wurtz RH (1995a) Saccade-related activity in monkey superior colliculus. I. Characteristics of burst and buildup cells. J Neurophysiol 73:2313-2333.

Munoz DP, Wurtz RH (1995b) Saccade-related activity in monkey superior colliculus. II. Spread of activity during saccades. J Neurophysiol 73:2334-2348.

Munoz DP, Guitton D, Pélisson D (1991) Control of orienting gaze shifts by the tectoreticulospinal system in the head-free cat. III. Spatiotemporal characteristics of phasic motor discharges. J Neurophysiol 66:1642-1666.

Munoz DP, Waitzman DM, Wurtz RH (1996) Activity of neurons in monkey superior colliculus during interrupted saccades. J Neurophysiol 75:2562-2580.

Ottes FP, Van Gisbergen JA, Eggermont JJ (1986) Visuomotor fields of the superior colliculus: a quantitative model. Vision Res 26:857-873.

Pélisson D, Prablanc C, Urquizar C (1988) Vestibuloocular reflex inhibition and gaze saccade control characteristics during eye-head orientation in humans. J Neurophysiol 59:997-1013.

Pélisson D, Guitton D, Goffart L (1995) On-line compensation of gaze shifts perturbed by micro-stimulation of the superior colliculus in the cat with unrestrained head. Exp Brain Res 106:196-204.

Port NL, Sommer MA, Wurtz RH (2000) Multielectrode evidence for spreading activity across the superior colliculus movement map. J Neurophysiol 84:344-357.

Quaia C, Lefèvre P, Optican LM (1999) Model of the control of saccades by superior colliculus and cerebellum. J Neurophysiol 82:999-1018.

Rodgers CK, Munoz DP, Scott SH, Paré M (2006) Discharge properties of monkey tectoreticular neurons. J Neurophysiol 95:3502-3511.

Roucoux A, Guitton D, Crommelinck M (1980) Stimulation of the superior colliculus in the alert cat. II. Eye and head movements evoked when the head is unrestrained. Exp Brain Res 39:75-85.

Scudder CA, Kaneko CS, Fuchs AF (2002) The brainstem burst generator for saccadic eye movements: a modern synthesis. Exp Brain Res 142:439-462.

Soetedjo R, Kaneko CR, Fuchs AF (2002a) Evidence that the superior colliculus participates in the feedback control of saccadic eye movements. J Neurophysiol 87:679-695.

Soetedjo R, Kaneko CR, Fuchs AF (2002b) Evidence against a moving hill in the superior colliculus during saccadic eye movements in the monkey. J Neurophysiol 87:2778-2789.

Sparks DL (2004) Commands for coordinated eye and head movements in the primate superior colliculus. In: The superior colliculus: new approaches for studying sensorimotor integration (Hall WC, Moschovakis A, eds), pp 303-318. Boca Raton, FL: CRC.

Sylvestre PA, Cullen KE (2006) Premotor correlates of integrated feedback control for eye-head gaze shifts. J Neurosci 26:4922-4929.

Takahashi M, Sugiuchi Y, Izawa Y, Shinoda Y (2005) Commissural excitation and inhibition by the superior colliculus in tectoreticular neurons projecting to omnipause neuron and inhibitory burst neuron regions. J Neurophysiol 94:1707-1726.

Tomlinson RD (1990) Combined eye-head gaze shifts in the primate. III. Contributions to the accuracy of gaze saccades. J Neurophysiol 64:1873-1891.

Walton MM, Bechara B, Gandhi NJ (2007) Role of the primate superior colliculus in the control of head movements. J Neurophysiol 98:20222037. 